\title{
Cigarette smoke mediates epigenetic repression of miR-487b during pulmonary carcinogenesis
}

\author{
Sichuan Xi, ${ }^{1}$ Hong Xu, ${ }^{2}$ Jigui Shan, ${ }^{3}$ Yongguang Tao, ${ }^{2}$ Julie A. Hong, ${ }^{1}$ Suzanne Inchauste, ${ }^{1}$ \\ Mary Zhang, ${ }^{1}$ Tricia F. Kunst, ${ }^{1}$ Leandro Mercedes, ${ }^{1}$ and David S. Schrump ${ }^{1}$
}

\begin{abstract}
${ }^{1}$ Thoracic Oncology Section, Surgery Branch, Center for Cancer Research, National Cancer Institute, Bethesda, Maryland, USA. 2Laboratory of Cancer Prevention, National Cancer Institute, Frederick, Maryland, USA. ${ }^{3}$ Advanced Biomedical Computing Center, SAIC-Frederick, National Cancer Institute, Frederick, Maryland, USA.
\end{abstract}

\begin{abstract}
MicroRNAs are critical mediators of stem cell pluripotency, differentiation, and malignancy. Limited information exists regarding microRNA alterations that facilitate initiation and progression of human lung cancers. In this study, array techniques were used to evaluate microRNA expression in normal human respiratory epithelia and lung cancer cells cultured in the presence or absence of cigarette smoke condensate (CSC). Under relevant exposure conditions, CSC significantly repressed miR-487b. Subsequent experiments demonstrated that miR-487b directly targeted SUZ12, BMI1, WNT5A, MYC, and KRAS. Repression of miR-487b correlated with overexpression of these targets in primary lung cancers and coincided with DNA methylation, de novo nucleosome occupancy, and decreased H2AZ and TCF1 levels within the miR-487b genomic locus. Deoxyazacytidine derepressed miR-487b and attenuated CSC-mediated silencing of miR-487b. Constitutive expression of miR-487b abrogated Wnt signaling, inhibited in vitro proliferation and invasion of lung cancer cells mediated by CSC or overexpression of miR-487b targets, and decreased growth and metastatic potential of lung cancer cells in vivo. Collectively, these findings indicate that miR-487b is a tumor suppressor microRNA silenced by epigenetic mechanisms during tobacco-induced pulmonary carcinogenesis and suggest that DNA demethylating agents may be useful for activating miR-487b for lung cancer therapy.
\end{abstract}

\section{Introduction}

In addition to irreversible mutations involving oncogenes and tumor suppressor genes as well as potentially reversible epigenomic alterations mediated by DNA methylation and histone modifications, perturbations of microRNA (miRNA) expression have been increasingly implicated in the pathogenesis of human cancers (1-3). These mature noncoding RNAs typically function as components of miRNA-containing, RNA-induced silencing complexes (miR-RISC) that interact in the cytoplasm with complementary mRNA sequences primarily in $3^{\prime}$ UTRs, and less commonly in ORFs and 5' UTRs, to initiate transcript degradation or inhibit translation (reviewed in refs. 1, 3, 4). In addition, some miRNAs associate with RISC components in the nucleus, and together with chromatic remodeling complexes, bind to promoter regions to either repress or activate transcription $(5,6)$. Approximately $60 \%$ of all mRNAs are potential miRNA targets. To date, more than 1,000 miRNAs have been identified in humans, each of which targets multiple functionally related genes, thereby regulating complex signaling networks $(2,3)$.

Numerous miRNAs, which function either as oncogenes (oncomirs) or tumor suppressors, are aberrantly expressed in human lung cancers $(7,8)$, the vast majority of which are directly attributable to cigarette smoking (9). For example, upregulation of miR-31 in lung cancer cells and normal respiratory epithelia following cigarette smoke exposure mediates repression of a variety of Wnt antagonists, such as DKK1 and SFRP1, resulting in activation of stem cell signaling and enhanced proliferation, invasion,

Conflict of interest: The authors have declared that no conflict of interest exists. Citation for this article: J Clin Invest. 2013;123(3):1241-1261. doi:10.1172/JCI61271. and tumorigenicity of lung cancer cells (10); activation of miR-31 also enhances malignant phenotype of lung cancer cells by inhibiting expression of LATS2 and PPP2R2A (11). Overexpression of miR-26a increases migration and invasion of lung cancer cells via repression of PTEN and upregulation of VEGF, Twist, $\beta$-catenin, and MMP-2 (12). Downregulation of miR-200 and miR-205 induces epithelial-to-mesenchymal transition (EMT) and stem cell phenotype in human bronchial epithelial cells (HBECs) exposed to tobacco carcinogens (13). Downregulation of miR-34a by the mesenchymal-associated repressor ZEB-1 enhances prometastatic cytoskeletal alterations in lung cancer cells (14). The fact that some miRNA alterations identified in lung cancers have also been detected in normal or cancer stem cells $(1,7,8,15,16)$ suggests that embryonic reprogramming of miRNA expression is a common theme of multistep pulmonary carcinogenesis.

Despite recent studies demonstrating miRNA expression profiles correlating with tumor histology as well as smoking status, response to therapy, and overall survival of patients with primary lung cancers (reviewed in ref. 8, 17), limited information is available regarding mechanisms by which miRNA alterations directly contribute to initiation and early progression of these malignancies. In the present study, we utilized an in vitro model system to examine miRNA alterations mediated by cigarette smoke condensate (CSC) in normal human respiratory epithelia and lung cancer cells derived from smokers as well as nonsmokers. Herein, we report that CSC mediates repression of miR-487b, thereby upregulating SUZ12, BMI1, WNT5A, MYC, and KRAS, resulting in increased proliferation, invasion, tumorigenicity, and metastatic potential of lung cancer cells. Downregulation of miR-487b and concomitant upregulation of these 5 targets were observed in 
primary lung cancers relative to paired adjacent normal lung tissues. These observations provide a direct mechanistic link between repression of a novel tumor suppressor miRNA and upregulation of putative mediators of cancer stem cell pluripotency during tobacco-induced human pulmonary carcinogenesis.

\section{Results}

Repression of miR-487b in cultured cells exposed to CSC. Preliminary PCR-array experiments were undertaken to examine the effects of CSC on miRNA expression in the panel of lung cancer cell lines and normal respiratory epithelia. This analysis suggested that CSC exposure modulated expression of numerous miRNAs, representative results pertaining to normal small airway epithelial cells (SAECs) and immortalized HBECs as well as Calu-6 and H841 lung cancer cells are depicted in Supplemental Figure 1; supplemental material available online with this article; doi:10.1172/ JCI61271DS1. Notably, CSC decreased expression of miR-487b, a relatively uncharacterized miRNA (18), in normal respiratory epithelia as well as lung cancer cells.

To further investigate this phenomenon, quantitative RT-PCR (qRT-PCR) experiments were performed to examine miR-487b expression in SAECs, HBECs, and Calu-6 and H841 cells cultured in normal medium (NM) with DMSO (control) or CSC with DMSO (CSC) for 5 days. Endogenous levels of miR-487b in untreated Calu- 6 and H841 lung cancer cells were significantly lower than those observed in SAECs and HBECs, which in turn were lower than those observed in normal human lung tissues (Figure 1 and Supplemental Figure 2); additional analysis revealed that 5-day CSC exposure downregulated miR-487b expression approximately 5.5- and 3.0-fold in SAECs and HBECs, respectively, relative to controls. Similar treatment decreased miR-487b expression approximately 7.6- and 2.7-fold in Calu-6 and H841 cells, respectively (Figure 1B). Time course experiments revealed downregulation of miR-487b in SAECs, HBECs, and Calu- 6 and H841 cells within 24 hours following initiation of CSC exposure, with expression continuing to decrease to levels nearly 100-fold lower than baseline over the next 3-4 days (Figure 1C). Additional qRT-PCR analysis revealed persistent repression of miR-487b 20 days following removal of CSC from culture medium, suggestive of reprogramming (Figure 1B).

Additional experiments were performed to determine whether nicotine could recapitulate the effects of CSC on miR-487b expression. Briefly, SAECs, HBECs, and Calu- 6 and H841 cells were cultured for 5 days in NM with or without nicotine. qRT-PCR experiments (Figure 1D) demonstrated that nicotine mediated dose-dependent repression of miR-487b in these cultured cells. Mass spectroscopy experiments (data not shown) revealed that the nicotine content in our CSCs was approximately $3.2 \mu \mathrm{g} / \mathrm{ml}$, which was well below the threshold for repression of miR-487b by nicotine. Furthermore, whereas 5-day CSC exposure mediated more than 90\% repression of miR-487b in cultured normal respiratory epithelia and lung cancer cells, pure nicotine only repressed miR- $487 \mathrm{~b}$ by $75 \%-80 \%$ relative to untreated controls even when used at concentrations far exceeding those in the condensates. These findings suggest that under conditions used for our experiments, CSC-mediated repression of miR-487b was not primarily attributable to nicotine.

Downregulation of miR-487b in primary lung cancer specimens. qRTPCR experiments were performed to examine miR-487b expression levels in a randomly selected panel of 51 primary lung cancers and adjacent histologically normal lung parenchyma (clinical and pathological data summarized in Table 1). As shown in Figure 1D and Supplemental Figure 3, miR-487b expression was significantly decreased (mean, 13.76-fold; range, 1.8-46.74 fold) in lung cancers relative to that in paired normal lung tissues $(P<0.01)$. The magnitude of miR-487b repression was greater in lung cancers from active and former smokers compared with never smokers (15.6-vs. 5.16-fold, respectively; $P<0.01$ ). Furthermore, miR-487b expression levels were significantly lower in histologically normal lung parenchyma from active or former smokers relative to never smokers; in fact, miR-487b expression in histologically normal lung tissues from smokers was significantly less than that observed in lung cancers from never smokers. Collectively, these data confirmed preliminary experiments demonstrating lower levels of miR-487b expression in lung cancer cells relative to cultured normal respiratory epithelia (Figure 1A and Supplemental Figure 2) and suggested that repression of miR-487b might be a biologically relevant phenomenon during human pulmonary carcinogenesis.

Effects of miR-487b on PRC components and Wnt signaling. Softwareguided analysis revealed several potential targets of miR-487b, including SUZ12 and BMI1, encoding core components of polycomb repressor complexes (PRC) 2 and 1, respectively, implicated in stem cell homeostasis and cancer $(19,20)$ as well as WNT5A, encoding a noncanonical Wnt ligand with diverse functions in normal respiratory epithelia and cancer $(21,22)$. To confirm these results, SAECs, HBECs, and Calu- 6 and H841 cells were transiently transfected with miR-487b precursor constructs. qRT-PCR experiments revealed approximately 3 - to 7 -fold increases in miR-487b levels in cells constitutively expressing miR-487b (miR-487b OEX) relative to vector controls (Figure 2A). Overexpression of miR-487b decreased SUZ12, BMI1, and WNT5A 2.92- to 6.99-fold, 6.33- to 11.42-fold, and 4.11- to 7.21-fold, respectively, in these 4 cell lines relative to vector controls (Figure $2 \mathrm{~B}$ ). These effects appeared somewhat more pronounced in lung cancer cells (Calu- 6 and H841), possibly due to lower levels of endogenous miR-487b and higher levels of SUZ12, BMI1, and WNT5A in these cells relative to SAECs and HBECs (data not shown).

Additional experiments were undertaken to determine whether depletion of endogenous miR-487b affected expression of SUZ12, $B M I 1$, and WNT5A in cultured normal respiratory epithelia and lung cancer cells. As shown in Figure 2C, miR-487b expression levels were reduced approximately 15 - to 20 -fold in SAECs, HBECs, and Calu- 6 and H841 cells transiently transfected with antisensemiR-487b oligos relative to respective control cells transfected with scrambled oligos. Knockdown of miR-487b increased expression of SUZ12, BMI1, and WNT5A in these cell lines (3.30- to 5.13-fold, 2.93- to 7.93-fold, and 2.16- to 7.98-fold, respectively; Figure 2D). Subsequent experiments revealed that overexpression of miR-487b significantly attenuated CSC-mediated increases in SUZ12, BMI1, and WNT5A in SAECs and HBECs as well as Calu-6 and H841 cells (Figure 2E); this phenomenon was particularly notable for SUZ12 and BMI1. Immunoblot analysis demonstrated that overexpression of miR-487b decreased SUZ12, BMI1, and WNT5A protein levels in SAECs and Calu-6 cells (Figure 2F; corresponding densitometry analysis summarized in Supplemental Figure 4). In contrast, knockdown of endogenous miR-487b increased SUZ12, BMI1, and WNT5A protein levels in SAECs, and to a somewhat lesser extent, in Calu- 6 cells. Collectively, these experiments suggested that miR-487b modulates SUZ12, BMI1, and WNT5A via post-transcriptional mechanisms in cultured normal respiratory epithelia and lung cancer cells. 
A

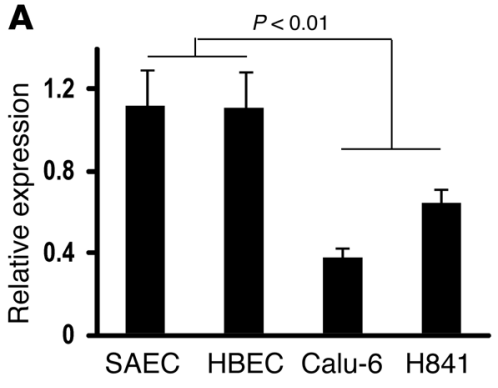

C

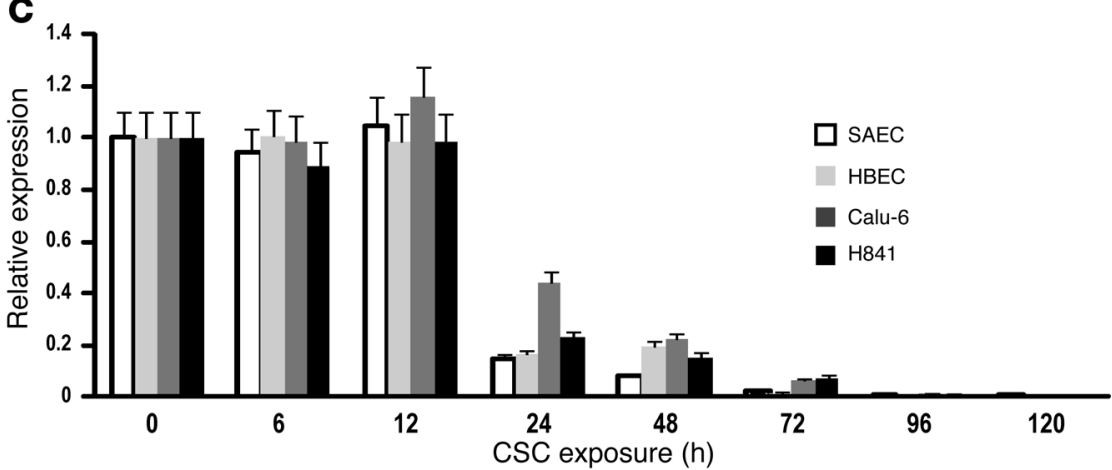

D

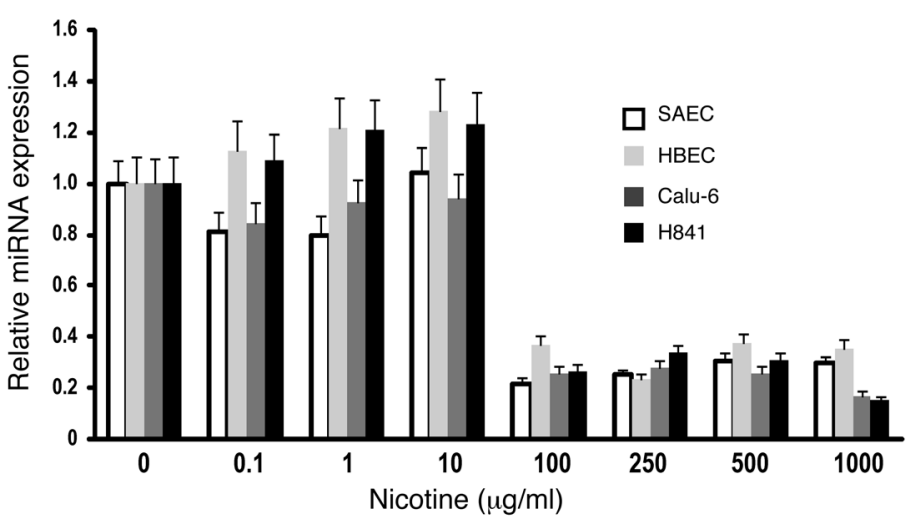

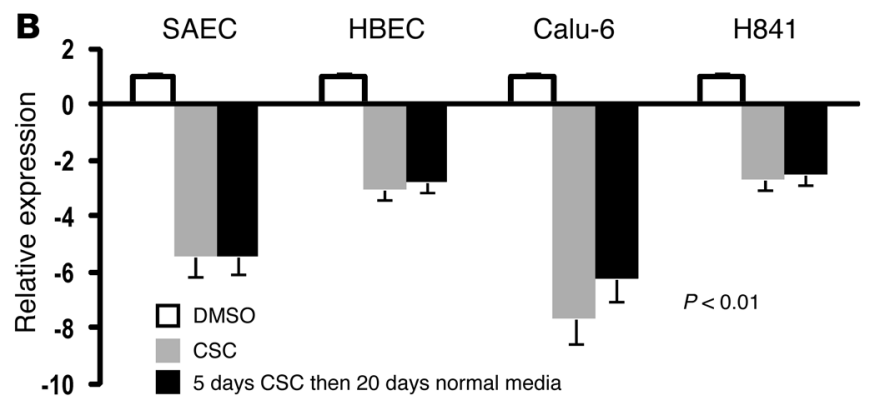

$\mathbf{E}$
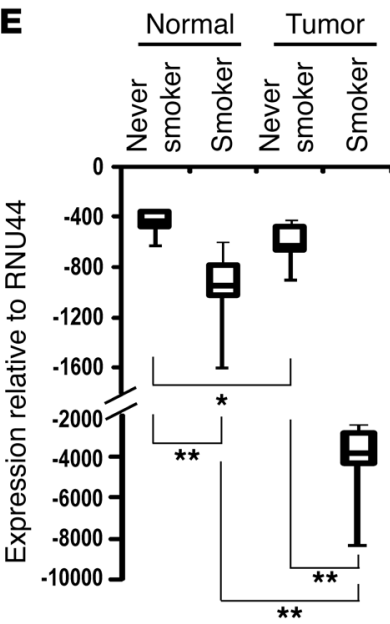

Figure 1

Expression of miR-487b in cultured cells as well as primary lung cancers and adjacent normal lung tissues. (A) qRT-PCR analysis of endogenous miR-487b expression normalized with control miRNA (RNU44) in SAECs, HBECs, Calu-6, and H841 cells. (B) qRT-PCR analysis demonstrating downregulation of miR-487b in cultured cells following 5-day CSC exposure. Repression of miR-487b persisted following discontinuation of CSC treatment. (C) qRT-PCR analysis demonstrating time-dependent repression of miR-487b in SAECs, HBECs, Calu-6, and H841 cells following CSC exposure. (D) qRT-PCR analysis demonstrating dose-dependent repression of miR-487b in lung cancer cells by nicotine, which leveled off with concentrations of $100 \mu \mathrm{g} / \mathrm{ml}$ or more following 5-day exposure. (E) qRT-PCR analysis of miR-487b expression in human lung cancers relative to paired adjacent normal lung tissues $(n=51)$. miR-487b levels were significantly lower in cancers relative to corresponding adjacent normal lung tissues. Furthermore, miR-487b levels were significantly more repressed in lung cancers from active/former smokers compared with those from never smokers. ${ }^{*} P<0.05 ;{ }^{* \star} P<0.01$.

Superarrays were used to further examine the effects of miR-487b on Wnt signaling in cultured normal respiratory epithelia and lung cancer cells. This analysis revealed that overexpression of miR-487b coincided with approximately 4.5- to 12 -fold downregulation of WNT5A, CTNNB1, LEF1, LRP6, and T cell factor 1 (TCF1) in SAECs as well as approximately 5- to 7-fold downregulation of WNT5A and WNT3A in Calu-6 cells (Supplemental Figure 5A). Furthermore, antagonists of Wnt signaling including DKK1, SFRP1, SFRP4, and WIF1 were upregulated approximately 4- to 12 -fold in SAECs and
Calu-6 cells (Supplemental Figure 5B). Subsequent qRT-PCR experiments confirmed that overexpression of miR-487b significantly upregulated DKK1, SFRP1, SFRP4, and WIF1 in normal SAECs as well as Calu-6, H841, and H358 lung cancer cells (Figure 2G).

miR-487b modulates Wnt antagonists by downregulation of PRC proteins. In light of our previous findings that CSC induces polycombmediated repression of DKK1 in normal respiratory epithelia and lung cancer cells (23), additional experiments were performed to determine whether modulation of DKK1, SFRP1, SFRP4, and WIF1 


\begin{tabular}{|c|c|c|c|}
\hline \multicolumn{2}{|l|}{ Characteristic } & \multirow{2}{*}{$\begin{array}{c}n \\
22\end{array}$} & \multirow{2}{*}{$\begin{array}{c}\text { Percentage } \\
43\end{array}$} \\
\hline Sex & Male & & \\
\hline & Female & 29 & 57 \\
\hline Age, yr & & 64 & \\
\hline \multirow[t]{4}{*}{ Histology } & Adenocarcinoma & 28 & 55 \\
\hline & Squamous cell carcinoma & 10 & 19.5 \\
\hline & Poorly differentiated carcinoma & 10 & 19.5 \\
\hline & Small cell carcinoma & 3 & 6 \\
\hline \multirow[t]{6}{*}{ Pathologic stage } & $\mathrm{TX}$ & 6 & 12 \\
\hline & T1-T2 & 35 & 68 \\
\hline & T3-T4 & 10 & 20 \\
\hline & $\mathrm{N}_{0}$ & 32 & 63 \\
\hline & $\mathrm{N}_{1}-\mathrm{N}_{2}$ & 17 & 33 \\
\hline & $\mathrm{N}_{3}$ & 2 & 4 \\
\hline \multirow[t]{2}{*}{ Smoking history } & Yes & 42 & 82 \\
\hline & No & 9 & 18 \\
\hline
\end{tabular}

Age reported as mean (range 46-88). Staging as determined by criteria of the International Association for the Study of Lung Cancer.

expression by miR-487b coincided with altered levels of polycomb proteins within the respective gene promoters. Quantitative ChIP analysis revealed significantly lower levels of SUZ12 and BMI1 (core components of initiation and maintenance PRC, respectively) within the DKK1, SFRP1, SFRP4, and WIF1 promoters in SAECs and Calu6 cells overexpressing miR-487b relative to respective vector control cells (Supplemental Figure 6, A and B). Collectively, these results suggest that miR-487b indirectly enhances expression of a variety of Wnt signaling antagonists in normal human respiratory epithelia and lung cancer cells by inhibiting expression of polycomb proteins that normally repress the respective genes encoding these antagonists.

SUZ12, BMI1, and WNT5A are direct targets of miR-487b. Additional studies were undertaken to determine whether miR-487b directly interacts with 3' UTRs of SUZ12, BMI1, and WNT5A. Briefly, AgoRNA cross-link immunoprecipitation (CLIP) techniques (10) were used to detect interaction of miR-487b with these potential targets in SAECs and Calu- 6 cells. Figure 3A depicts predicted miR-487b binding sites within the $3^{\prime}$ UTRs of SUZ12, BMI1, and WNT5A transcripts; single sites were identified in the $3^{\prime}$ UTRs of SUZ12 and $B M I 1$, whereas 2 potential binding sites were present in the WNT5A 3' UTR. RT-PCR analysis revealed that the AGO family antibody precipitated SUZ12, BMI1, and WNT5A transcripts in the cytoplasm of untreated SAECs and Calu- 6 cells (Figure 3B). Increased PCR products corresponding to miR-487b binding sites within the $3^{\prime}$ UTRs of SUZ12, BMI1, and WNT5A were observed in SAECs overexpressing miR-487b; this phenomenon was not observed in miR-487b-depleted cells. Quantitative CLIP analysis (Figure 3C) utilizing different primer sets confirmed results of gel-based CLIP experiments. For these experiments, $\beta$-actin served as the negative control, since its $3^{\prime}$ UTR contains no sequences targeted by miR-487b. Because miR- 31 has been found to directly target the $3^{\prime}$ UTR of DKK1 (10), immunoprecipitated RNA from SAECs and Calu- 6 cells overexpressing miR-31 served as a positive control for CLIP (Supplemental Figure 7).

Additional experiments were performed using pMiR-report vectors with WT or mutant 3' UTRs of SUZ12, BMI1, and WNT5A, transiently cotransfected with miR-487b precursor constructs or control vectors into SAECs and Calu- 6 cells. As shown in Figure 3D, luciferase activities of WT 3' UTRs of BMI1, SUZ12, and WNT5A were reduced approximately $40 \%-80 \%$ in cells overexpressing miR-487b relative to luciferase activities in cells transfected with control vectors. Mutation of the predicted miR-487b binding sites within these $3^{\prime}$ UTRs abolished repression mediated by miR-487b. Both potential binding sites in the WNT5A 3' UTR appeared to contribute to repression of WNT5A by miR-487b.

miR-487b targets MYC and KRAS. Software-assisted miRNA target screening predicted that MYC and KRAS (both of which have been implicated in initiation and progression of lung cancers) (24) are also potential targets of miR-487b (Figure 4A). As such, experiments were performed to determine whether miR-487b negatively regulates these protooncogenes in normal respiratory epithelia and lung cancer cells. Gel-based as well as quantitative CLIP experiments using different primer sets (Figure 4, B and C, respectively) demonstrated significant increases in RT-PCR products corresponding to 3' UTRs of MYC and KRAS in SAECs and Calu- 6 cells overexpressing miR-487b relative to respective vector controls; this phenomenon was abolished in these cells following knockdown of miR-487b. Subsequent qRT-PCR experiments (Figure 4D) demonstrated that overexpression of miR-487b significantly decreased MYC and KRAS expression in SAECs as well as Calu- 6 and H841 lung cancer cells. Conversely, knockdown of miR-487b increased MYC and KRAS levels in SAECs; however, depletion of miR-487b did not increase MYC and KRAS expression in Calu-6 or H841cells, possibly due to relatively low endogenous miR-487b levels in these cells. Immunoblot analysis (Figure 4E, with corresponding densitometry analysis depicted in Supplemental 8) demonstrated that overexpression of miR-487b coincided with decreased MYC and KRAS protein levels in SAECs as well as Calu- 6 and H-841 cells. Consistent with results of qRT-PCR experiments, knockdown of miR-487b increased MYC and KRAS protein levels above those observed in parental and vector control SAECs; this phenomenon was less evident in Calu-6 and H841 lung cancer cells, possibly due to very low endogenous miR-487b expression in these cells. Collectively, these experiments strongly suggest that miR-487b directly interacts with 3 ' UTRs of MYC and KRAS, thereby inhibiting expression of these protooncogenes in normal human respiratory epithelia and lung cancer cells.

Expression of miR-487b targets in primary lung cancers. qRT-PCR experiments were performed to evaluate expression levels of SUZ12, BMI1, WNT5A, MYC, and KRAS relative to miR-487b in primary lung cancer specimens and paired adjacent normal lung parenchyma. Results of this analysis are depicted in Figure 5. Expression levels of SUZ12, BMI1, WNT5A, MYC, and KRAS were significantly higher in the 51 primary lung cancers relative to paired adjacent normal lung parenchyma (Figure 5, A and B). The magnitude of miR-487b repression correlated with increased expression of all 5 of these targets in primary lung cancers (Figure 5, C and D, and Supplemental Figure 9).

Additional qRT-PCR experiments were performed to further examine expression levels of the aforementioned miR-487b targets in lung cancers relative to patient smoking status. As shown in Figure 5, E and F, SUZ12, BMI1, WNT5A, MYC, and KRAS expression levels were significantly higher in lung cancers from smokers/former smokers relative to never smokers; the magnitude of miR-487b repression correlated significantly with increased expression of all 5 miR-487b targets in these samples (Figure 5, G and H, and Supplemental Figure 10). Cigarette smoking history appeared to particularly influence the magnitude of the inverse correlation between miR-487b repression and upregulation of SUZ12, BMI1, and MYC in primary lung cancers (Figure $5 \mathrm{H}$ ). 

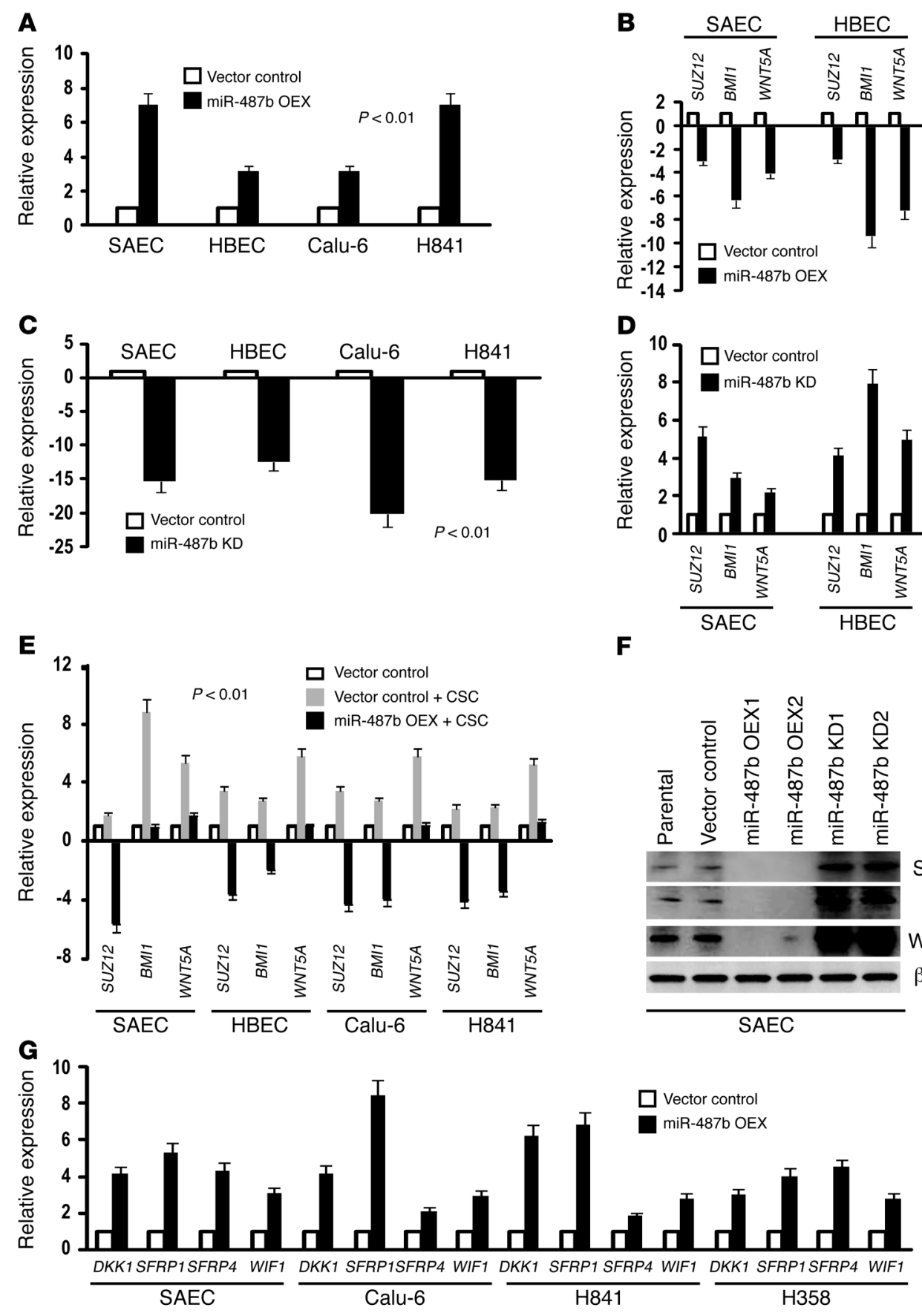

Figure 2

miR-487b negatively regulates SUZ12, BMI1, and WNT5A in cultured normal respiratory epithelial and lung cancer cells. (A) qRT-PCR analysis demonstrating miR-487b expression in normal respiratory epithelia and lung cancer cells constitutively expressing miR-487b (miR-487b OEX) relative to vector controls. (B) qRT-PCR analysis demonstrating that overexpression of miR-487b downregulates SUZ12, BMI1, and WNT5A in normal respiratory epithelia and lung cancer cells. (C) qRT-PCR analysis demonstrating decreased levels of endogenous miR-487b in SAECs, HBECs, Calu-6, and H841 cells following transient knockdown of miR-487b (miR-487b KD) relative to vector controls. (D) qRT-PCR analysis demonstrating that downregulation of miR-487b enhances levels of SUZ12, BMI1, and WNT5A expression in SAECs, HBECs, Calu-6, and H841 cells. (E) qRT-PCR analysis demonstrating that overexpression of miR-487b abrogates CSC-mediated increases in SUZ12, BMI1, and WNT5A in SAECs, HBECs, Calu-6, and H841 cells. (F) Immunoblot analysis of SUZ12, BMI1, and WNT5A expression in parental and vector control SAECs and Calu- 6 cells, as well as SAECs and Calu- 6 cells exhibiting constitutive overexpression or knockdown of miR-487b. Results of immunoblot experiments were consistent with qRT-PCR analysis (B and D). (G) Results of qRT-PCR analysis demonstrating that overexpression of miR-487b upregulates Wnt antagonists DKK1, SFRP1, SFRP4, and WIF1 in normal SAECs as well as Calu-6, H841, and H358 lung cancer cells. 
A
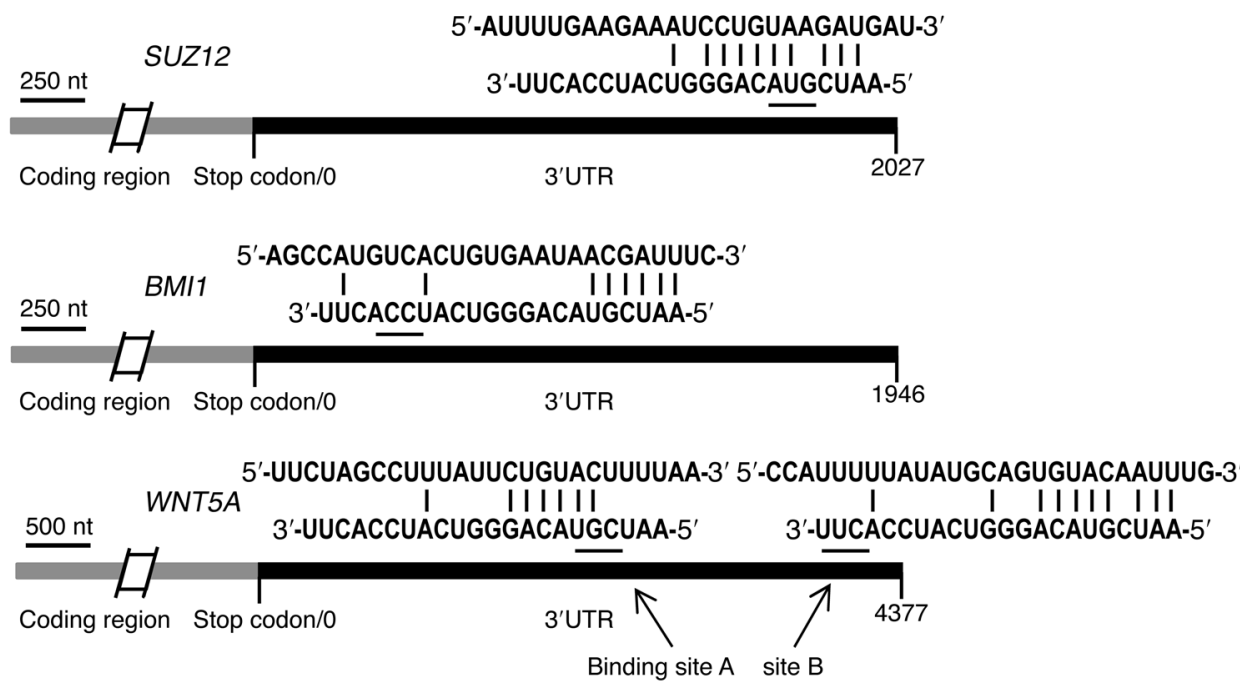

B

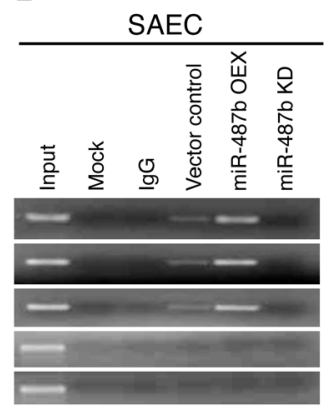

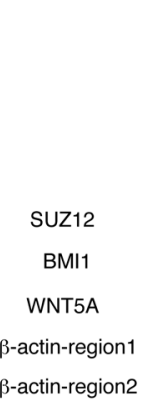

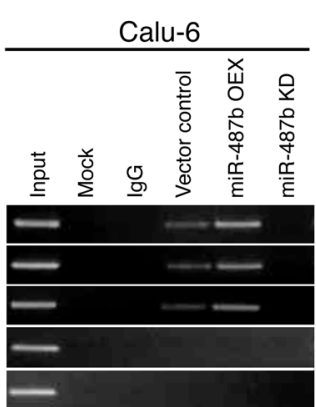

C

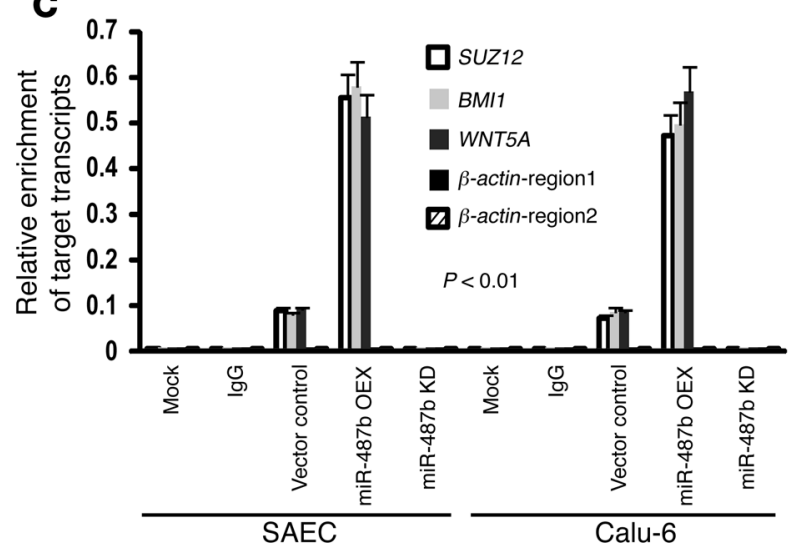

D $3^{\prime}$ UTR-

SUZ12

BMl1
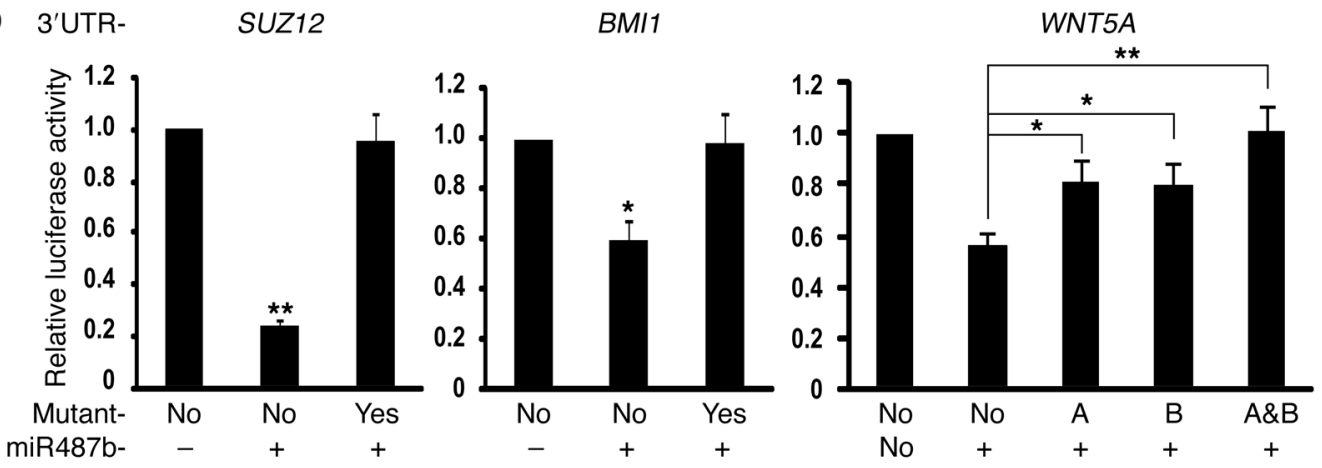

Figure 3

SUZ12, BMI1, and WNT5A are direct targets of miR-487b. (A) Putative target sites of miR-487b within SUZ12, BMI1, and WNT5A 3' UTRs (top, middle, and bottom, respectively). (B and C) Ago-CLIP (B) and quantitative Ago-CLIP (C) analyses of interactions of miR-487b with SUZ12, $B M I 1$, and WNT5A 3' UTRs in SAECs and Calu-6 cells. Overexpression of miR-487b significantly increased precipitation of SUZ12, BMI1, and WNT5A, indicating direct interaction of miR-487b with the respective $3^{\prime}$ UTRs. (D) Luciferase reporter assays demonstrating that overexpression of miR-487b decreases luciferase activities of WT but not mutant 3' UTRs of SUZ12, BMI1, and WNT5A in SAECs. For WNT5A, miR-487b-binding sites $A$ and/or $B$ were mutated; mutation of both miR-487b binding sites was more effective than single mutations in abrogating the effect of miR-487b. ${ }^{\star} P<0.05$; ${ }^{\star \star} P<0.01$.

miR-487b is a tumor suppressor in buman lung cancer cells. Additional experiments were undertaken to examine the effects of miR-487b on proliferation, invasion, and tumorigenicity of lung cancer cells. First, in vitro growth assays were performed using Calu-6, H841, and $\mathrm{H} 358$ cells following constitutive overexpression or depletion of miR-487b. These cell lines were chosen because they exhibit reliable tumorigenicity in athymic nude mice. As shown in Figure 6A, constitutive overexpression of miR-487b decreased proliferation of Calu- 6 and H841 cells by 33\%-50\% relative to respective vector controls; in contrast, knockdown of endogenous miR-487b enhanced 
A

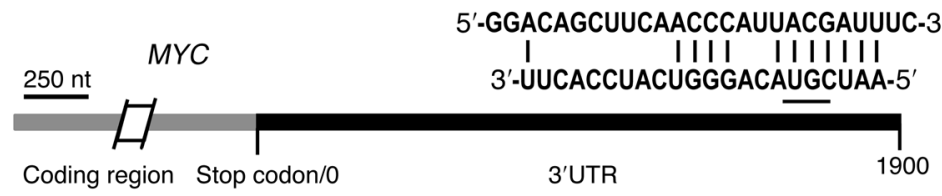

B
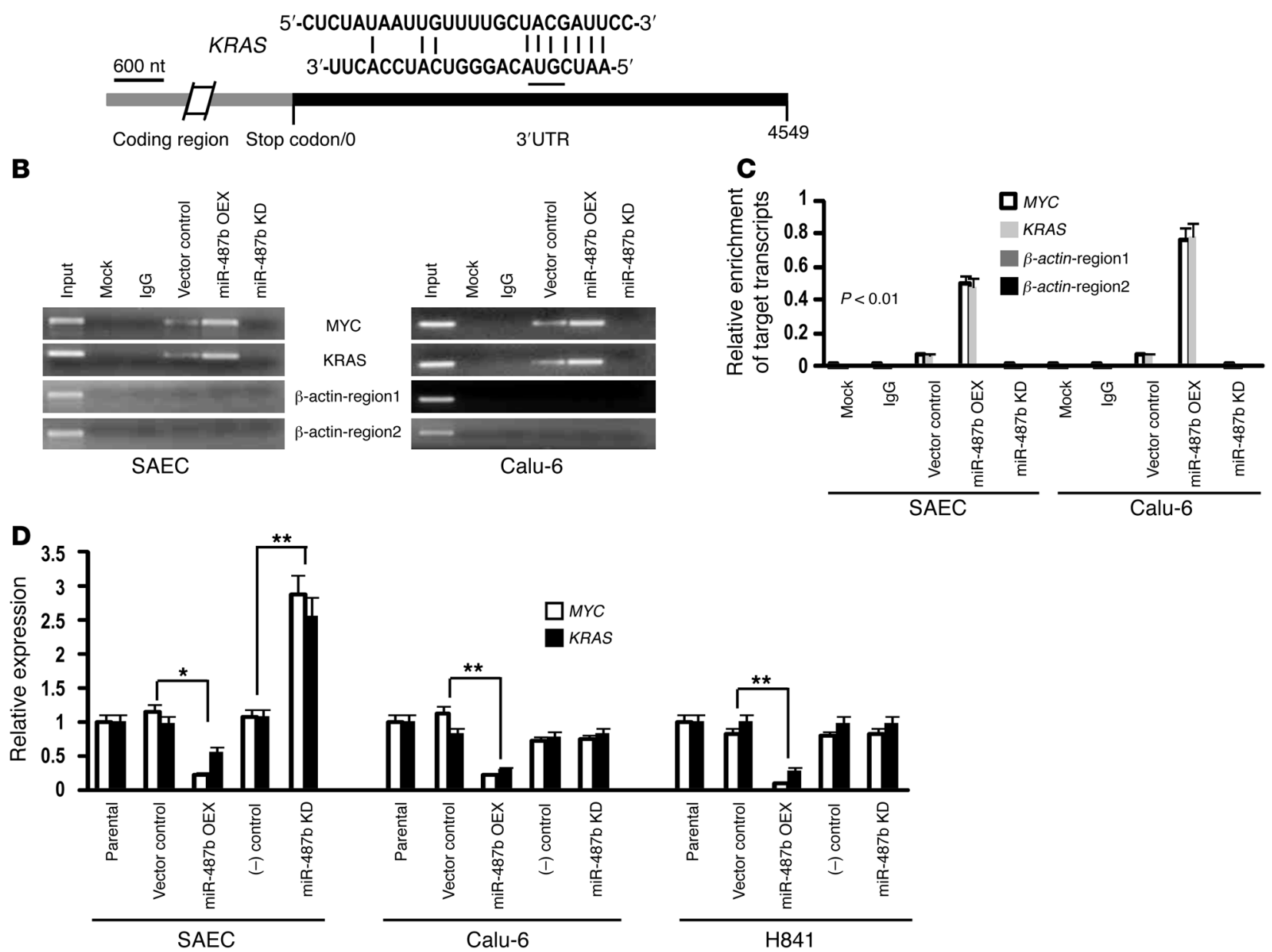

$\mathbf{E}$

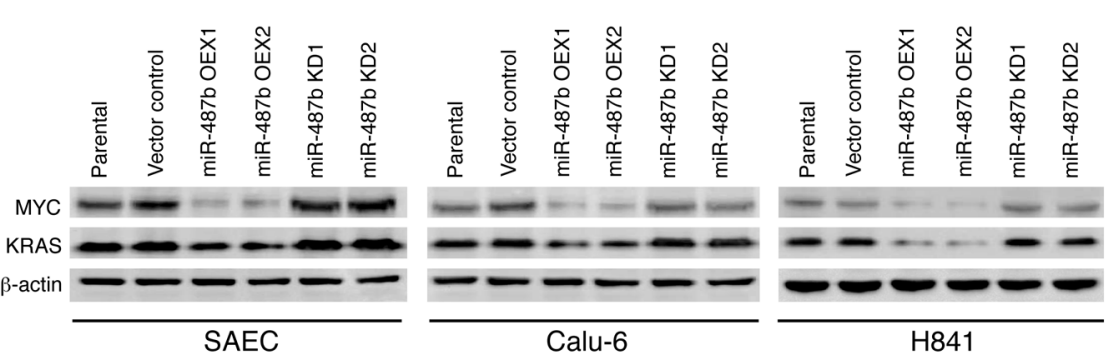

Figure 4

miR-487b targets MYC and KRAS. (A) Putative binding sites of miR-487b within MYC and KRAS 3' UTRs (upper and lower panels, respectively). (B and C) Ago-CLIP (B) and quantitative Ago-CLIP (C) analyses depicting direct interaction of miR-487b with $3^{\prime}$ UTRs of MYC and KRAS in SAECs and Calu- 6 cells. (D) qRT-PCR analysis of MYC and KRAS expression in parental SAECs, Calu-6, or H841 cells, vector controls, or corresponding cells exhibiting overexpression or knockdown of miR-487b. ${ }^{*} P<0.05$; ${ }^{* *} P<0.01$. (E) Immunoblot analysis of MYC and KRAS expression in SAECs and Calu- 6 and H841 cells following overexpression or knockdown of miR-487b. Overexpression of miR-487b decreased MYC and KRAS levels in all 3 lines. Knockdown of miR-487b increased MYC and KRAS levels above that of controls in SAECs and, to a lesser extent, in Calu- 6 and $\mathrm{H} 841$ cells. 

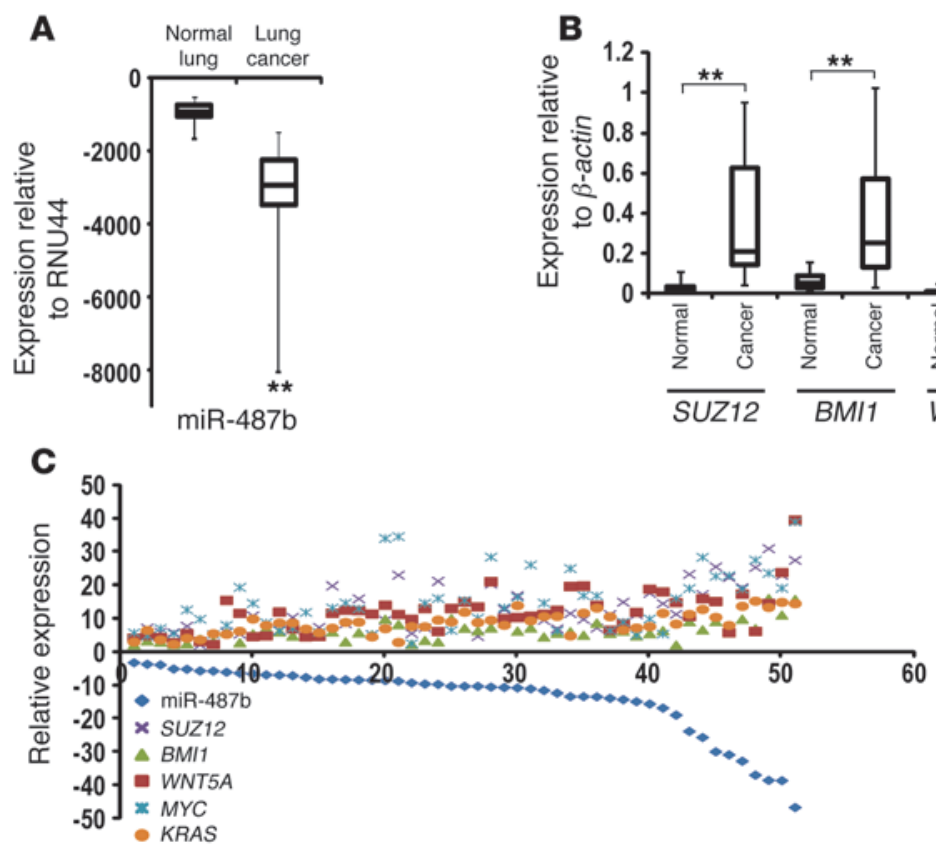

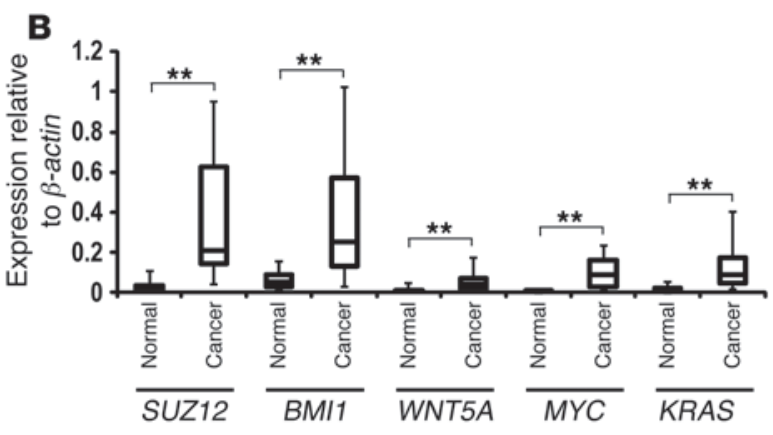

D

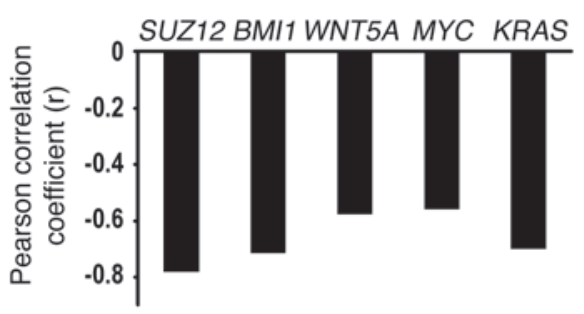

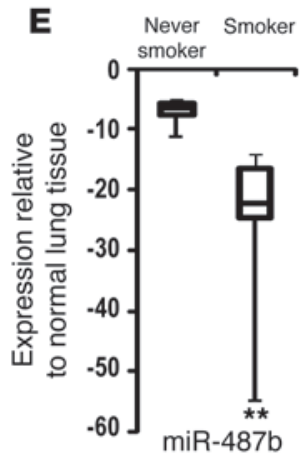

G

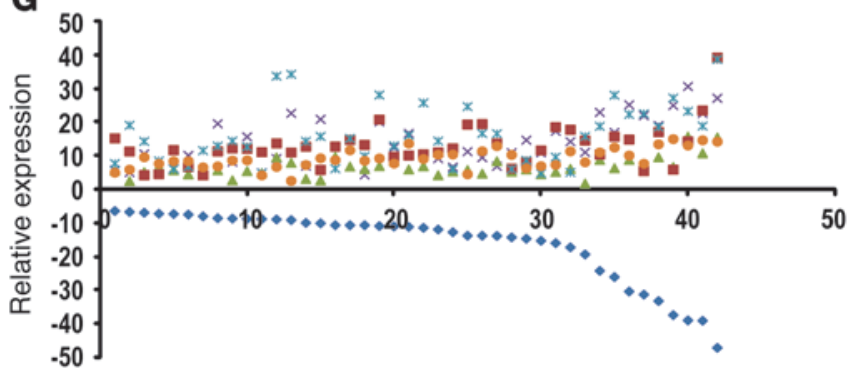

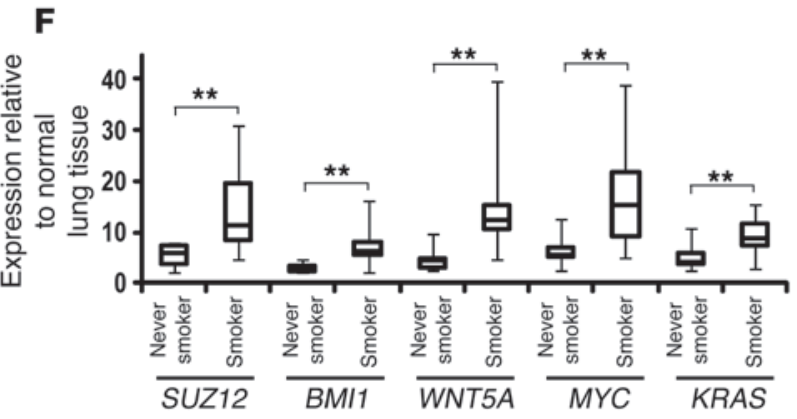

H

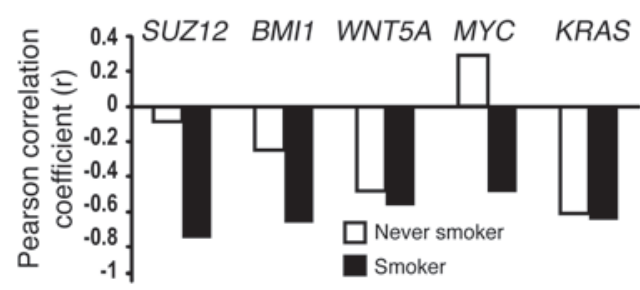

Figure 5

Reverse correlation between miR-487b and its 5 targets relative to smoking history of patients. (A) qRT-PCR analysis of miR-487b expression in 51 lung cancer specimens relative to paired adjacent normal lung tissues. (B) qRT-PCR analysis demonstrating increased SUZ12, BMI1, WNT5A, MYC, and KRAS expression levels in lung cancers relative to paired adjacent normal lung tissues. (C and $\mathbf{D})$ Correlation patterns (C) and corresponding correlation coefficient values (D) between miR-487b and its targets in tumor specimens relative to paired adjacent normal lung tissues $(n=51)$. (E) qRT-PCR analysis of miR-487b expression in lung cancers from smokers/former smokers versus never smokers. (F) qRT-PCR analysis demonstrating increased SUZ12, BMI1, WNT5A, MYC, and KRAS expression levels in lung cancers from smokers/former smokers relative to never smokers. (G) Correlation patterns between miR-487b and its targets in 42 lung cancers from smokers. (H) Corresponding coefficient values between miR-487b repression and upregulation of 5 targets in 9 lung cancers from never smokers and 42 lung cancers from smokers/former smokers. ${ }^{* \star} P<0.01$. 
A

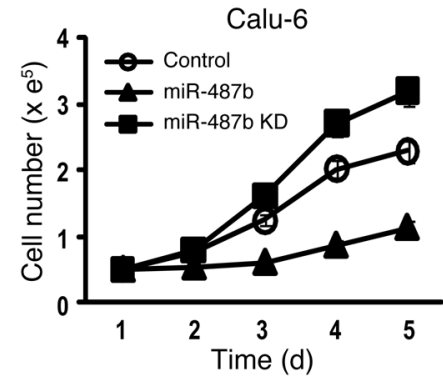

C

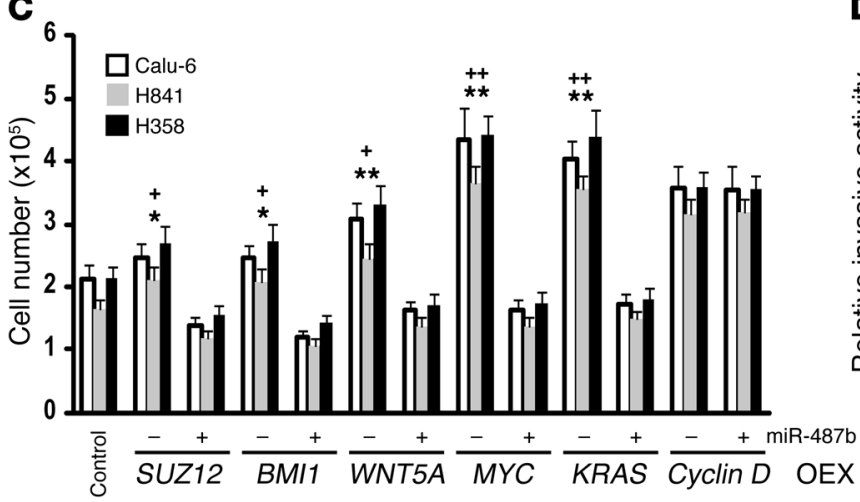

$\mathrm{H} 841$

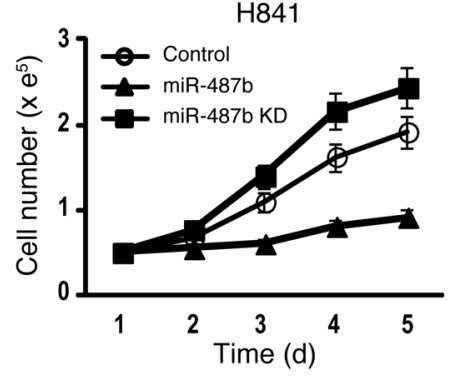

D
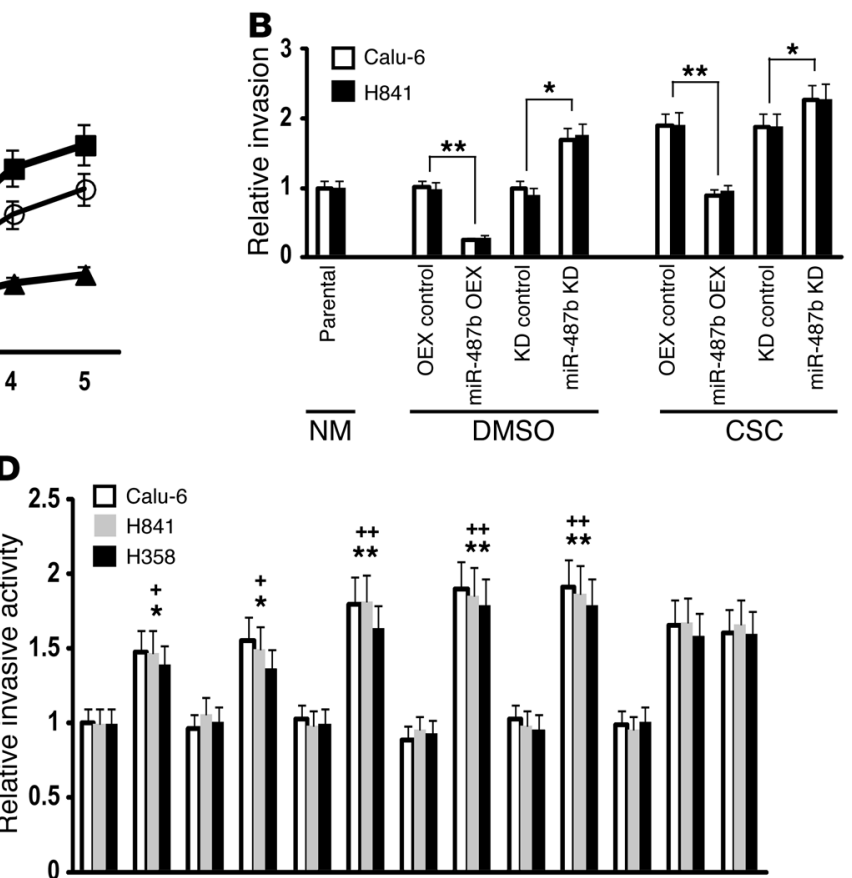

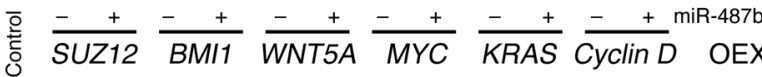

$\mathbf{E}$

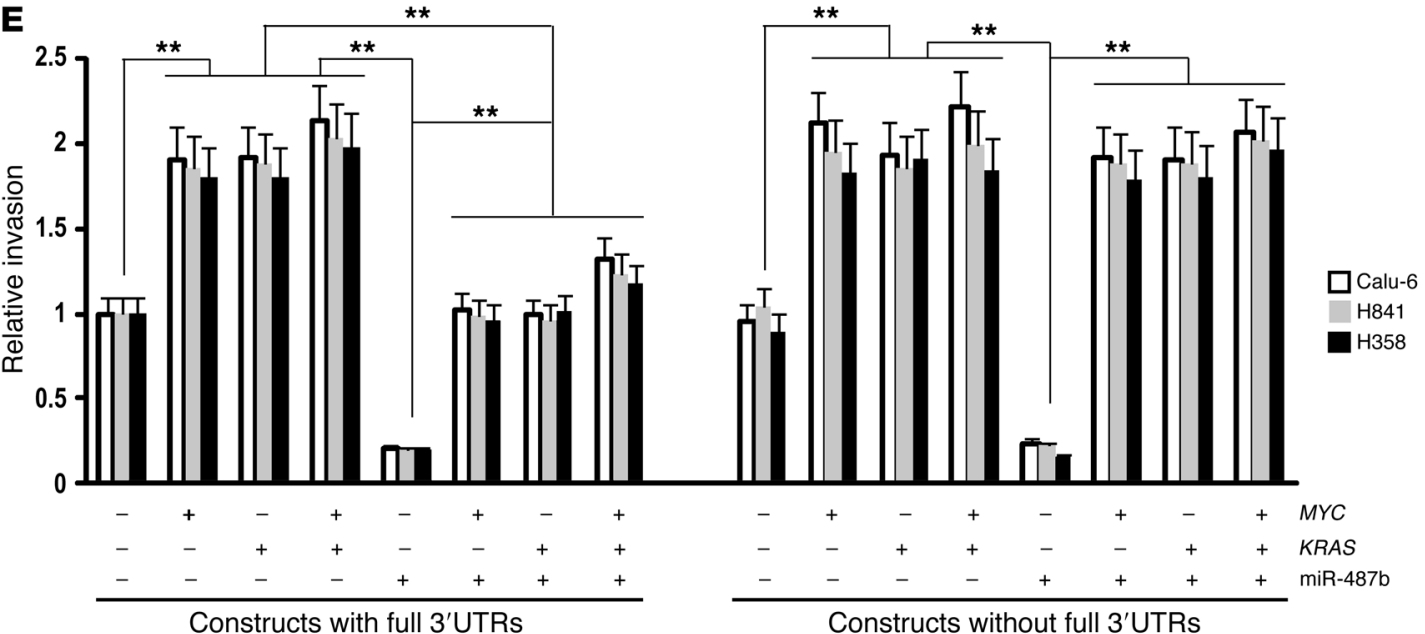

Figure 6

miR-487b functions as a tumor suppressor in lung cancer cells. (A) Effects of miR-487b expression on in vitro proliferation of Calu-6 and H841 lung cancer cells. (B) Matrigel invasion assays demonstrating that overexpression of miR-487b inhibited, whereas knockdown of miR-487b enhanced, invasion of Calu- 6 and H841 lung cancer cells. miR-487b significantly inhibited CSC-induced invasion of lung cancer cells. The effects of miR-487b knockdown on invasion of DMSO-treated cells were comparable to these observed in vector controls exposed to CSC. ${ }^{*} P<0.05$; ${ }^{\star \star} P<0.01$. (C) Cell count assays demonstrating effects of miR-487b on proliferation of lung cancer cells overexpressing SUZ12, BMI1, WNT5A, MYC, or KRAS. miR-487b had no effects on proliferation induced by cyclin $D 1$, which is not targeted by miR-487b. ${ }^{+} P<0.05$; ${ }^{++} P<0.01$ vs. control. ${ }^{\star} P<0.05$; ${ }^{* *} P<0.01$ vs. miR-487b. (D) Matrigel assays demonstrating effects of miR-487b on invasive potential of lung cancer cells following overexpression of the $5 \mathrm{miR}-487$ targets. miR-487b had no effects on invasion induced by cyclin $\mathrm{D} 1 .{ }^{+} P<0.05 ;{ }^{++} P<0.01 \mathrm{vs}$. control. ${ }^{*} P<0.05$; ${ }^{* *} P<0.01$ vs miR-487b. (E) Specificity of effects of miR-487b expression on invasion potential of lung cancer cells mediated by $M Y C$ and $K R A S$. miR-487b failed to inhibit invasion induced by overexpression of $M Y C$ and KRAS lacking full 3' UTR sequences.

proliferation of these cancer cells by approximately $30 \%$. Virtually identical results were observed following overexpression or knockdown of miR-487b in H358 lung cancer cells (data not shown).

Matrigel assays were performed to examine the effects of miR-487b on invasion potential of lung cancer cells. Constitutive overexpres- sion of miR-487b significantly decreased, whereas knockdown of miR-487b increased invasion, respectively, of lung cancer cells (representative results pertaining to Calu- 6 and H841 cells are depicted in Figure 6B). CSC exposure significantly increased invasion of vector control cells, the magnitude of which was similar to that 

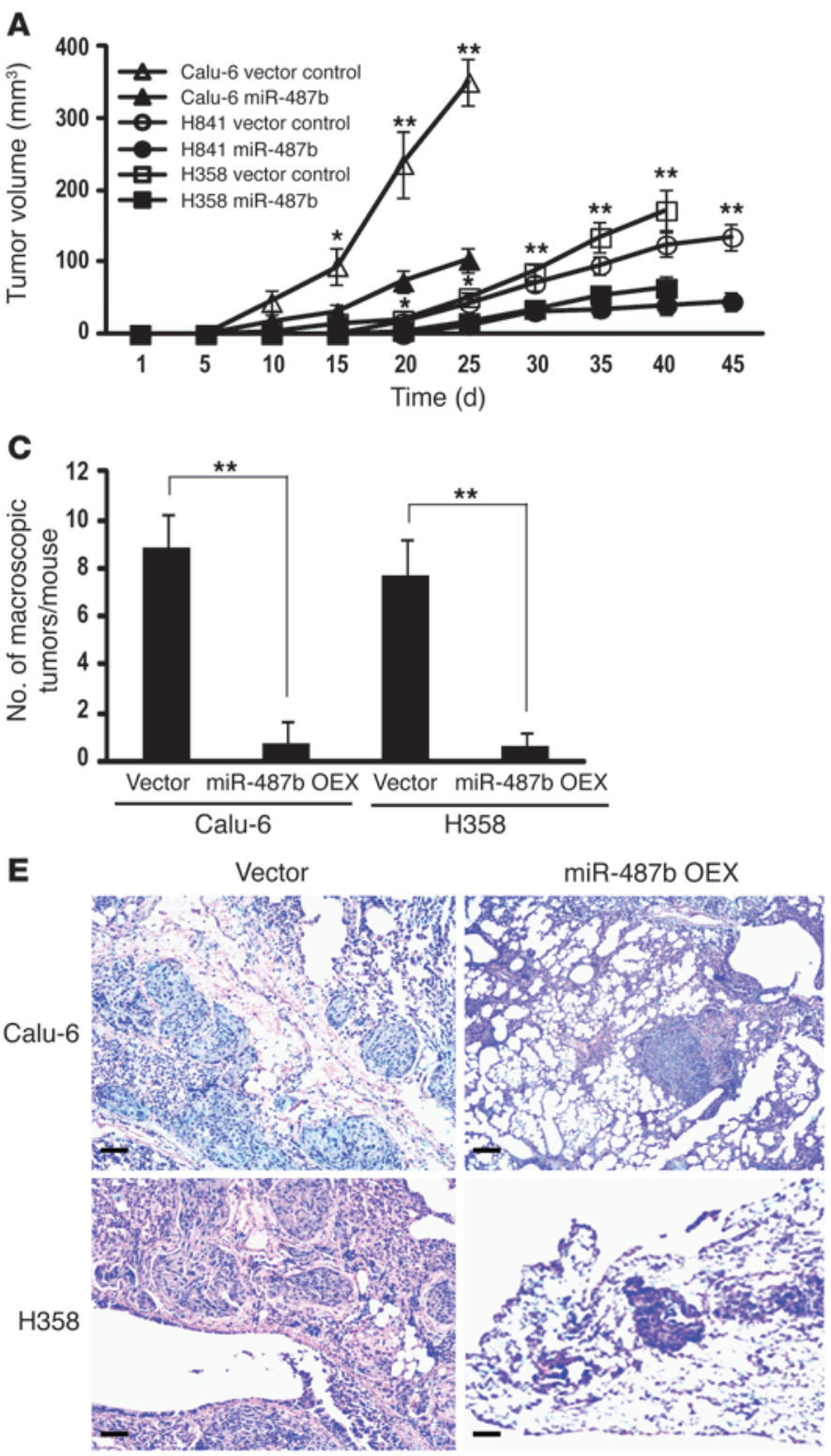
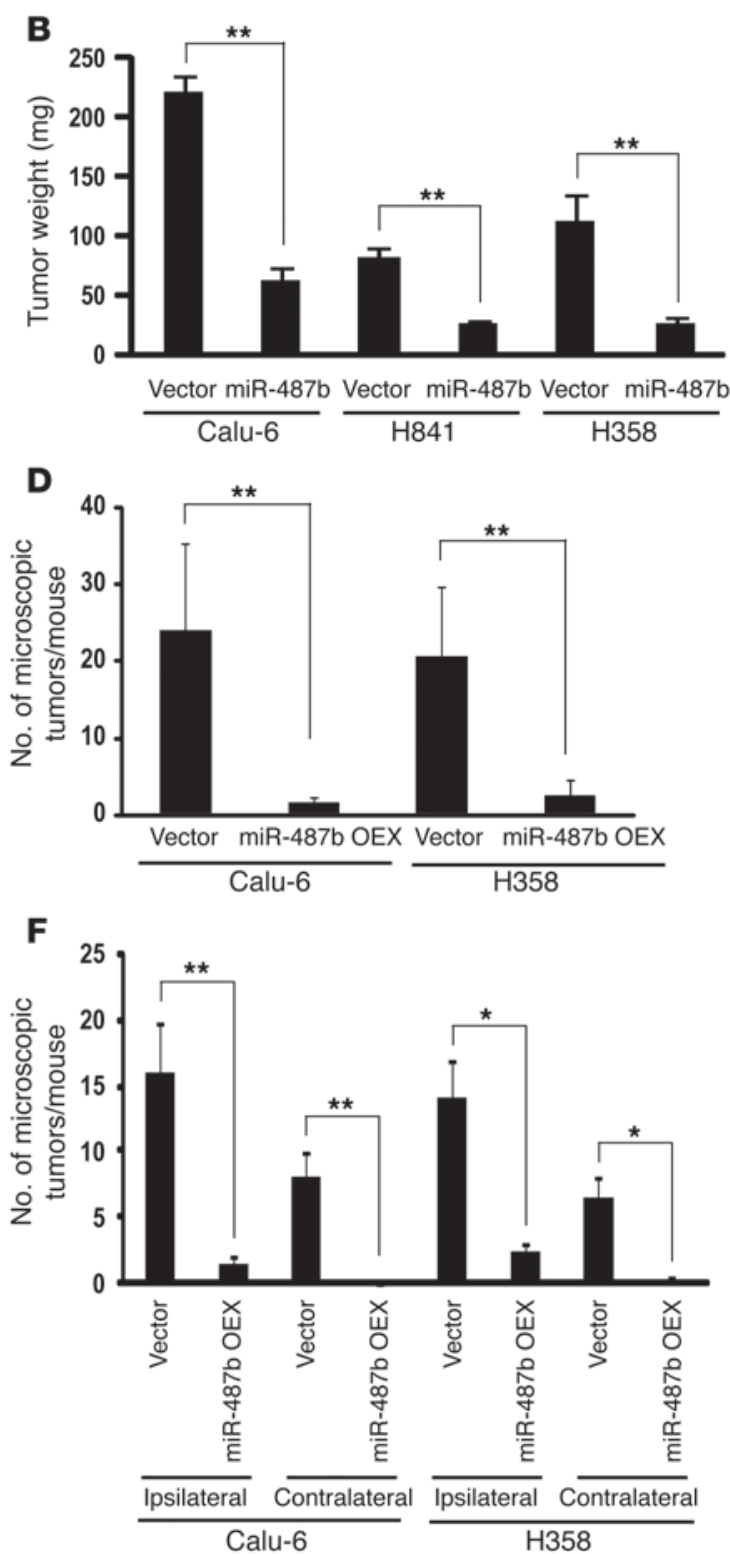

Figure 7

Effects of miR-487b on growth and metastatic potential of lung cancer cells in vivo. (A) Growth of Calu-6, H841, and H358 subcutaneous xenografts in nude mice. Volumes of xenografts derived from Calu-6, H841, or H358 cells overexpressing miR-487b were significantly smaller than control xenografts. (B) Tumor masses from Calu-6, H841, and H358 xenografts. miR-487b overexpression significantly decreased average mass of tumor xenografts. (C) Quantitation of macroscopic pulmonary nodules derived from Calu-6 or H358 cells stably expressing miR-487b or control vectors. (D) Quantitation of total microscopic pulmonary tumors following orthotopic injection of Calu-6 or H358 cells stably expressing miR-487b or control vectors. (E) Representative microscopic images (H\&E staining) of intrapulmonary tumors from Calu-6 or H358 cells stably expressing $\mathrm{miR}-487 \mathrm{~b}$ or control vectors. Expression of miR-487b decreased invasion of lung cancer cells in vivo. Scale bars: $50 \mathrm{~mm}$. Original magnification, $\times 100$. (F) Quantitation of ipsilateral and contralateral microscopic pulmonary tumors following orthotopic injection of Calu- 6 or H358 cells stably expressing miR-487b or control vectors. ${ }^{*} P<0.05 ;{ }^{* *} P<0.01$.

observed in lung cancer cells following knockdown of miR-487b. Overexpression of miR-487b inhibited CSC-mediated invasion by approximately $50 \%$ relative to control cells. Knockdown of miR-487b only modestly increased invasion of DMSO-treated (control) Calu- 6 and H841 cells; this phenomenon appeared to be less pronounced following CSC exposure, suggesting that the effects of miR-487b repression and CSC treatment were mediated, at least in part, by redundant mechanisms.
Additional experiments were performed to examine the relative effects of overexpression of SUZ12, BMI1, WNT5A, MYC, or KRAS on proliferation and invasion of lung cancer cells. Representative results pertaining to Calu-6 and H841 cells are depicted in Figure 6, $\mathrm{C}$ and $\mathrm{D}$. Whereas overexpression of any of the 5 potential targets of miR-487b significantly increased proliferation of lung cancer cells relative to vector controls, the effects of overexpression of $M Y C$ or KRAS were particularly dramatic. A similar phenomenon 
A

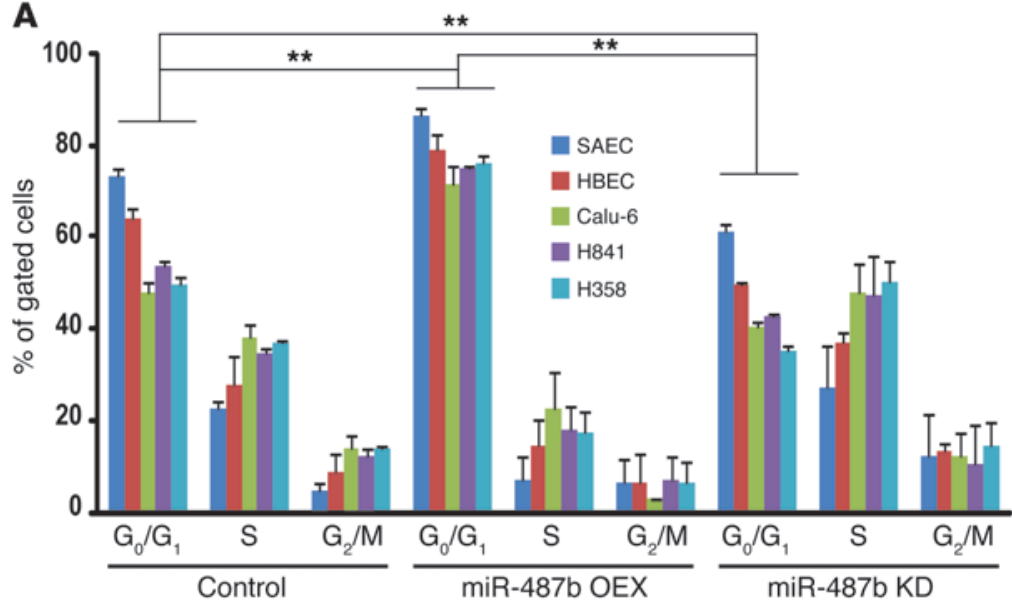

B

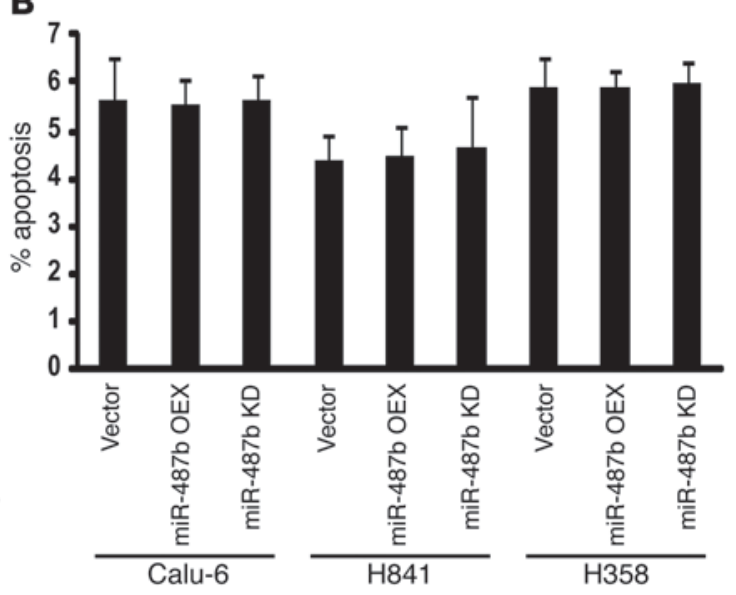

C

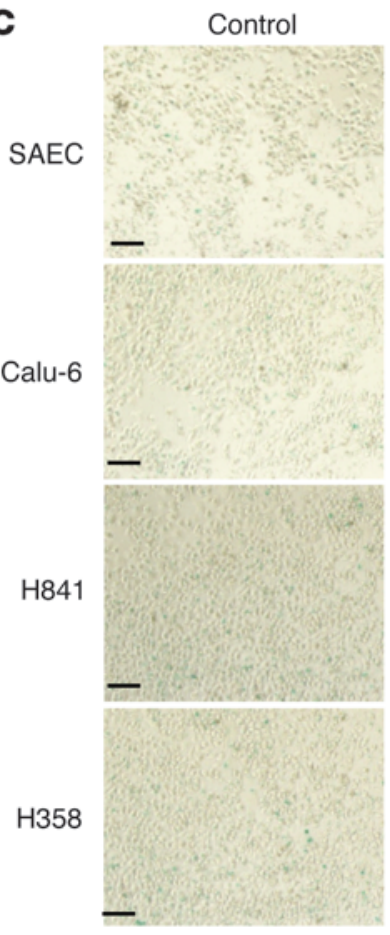

miR-487b OEX

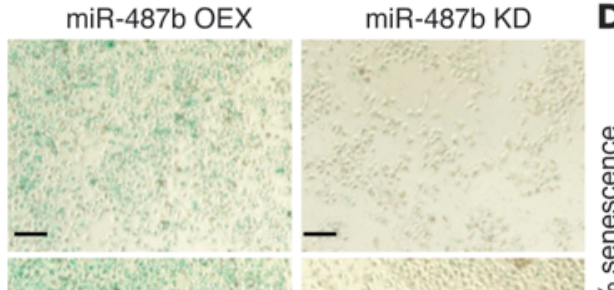

D

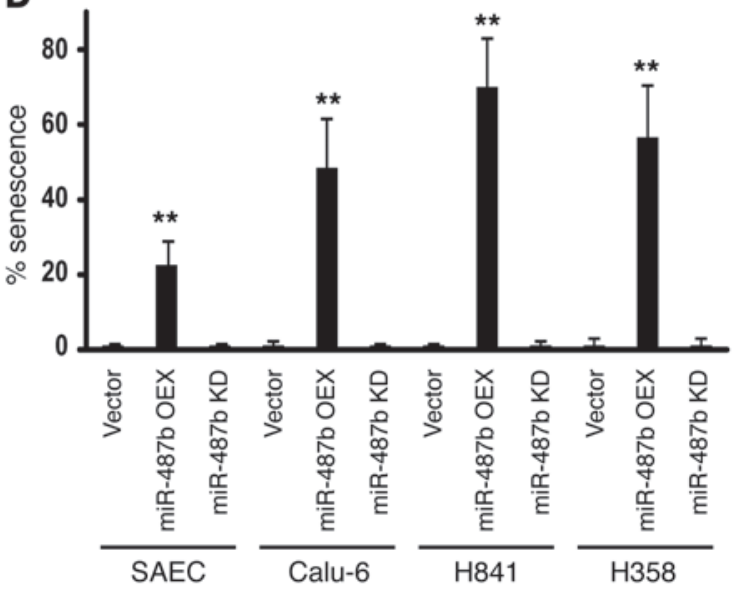

\section{Figure 8}

miR-487b inhibits cellular proliferation via cell-cycle arrest and senescence but not apoptosis in normal respiratory epithelia and lung cancer cells. (A) Flow cytometry analysis demonstrating that miR-487b overexpression significantly impairs progression of SAECs, HBECs, Calu-6, $H 841$, and $H 358$ cells from $G_{0} / G_{1}$ into $S$ and $G_{2} / M$ phases. Endogenous depletion of miR-487b enhanced cell-cycle progression in these cells. Data are represented as percentage of gated cells \pm SD. (B) In vitro apoptosis assay demonstrating no change in apoptosis index in lung cancer cells exhibiting overexpression or knockdown of miR-487b. (C) Representative microscopic images (senescence staining) of SAECs, Calu-6, $\mathrm{H} 841$, and H358 cells exhibiting overexpression or depletion of miR-487b. Scale bars: $50 \mathrm{~mm}$. Original magnification, $\times 100$. (D) Quantitation of miR-487b-induced cellular senescence in normal respiratory epithelia and lung cancer cells. Ectopic expression of miR-487b significantly increased percentage of senescence in these cells. However, depletion of miR-487b did not affect senescence in these cells. Data are represented as percentage of cells $\pm \mathrm{SD}$. ${ }^{\star \star} P<0.01$.

was observed regarding invasion of lung cancer cells following overexpression of WNT5A, BMI1, SUZ12, MYC, and KRAS; WNT5A appeared to enhance invasion more than proliferation of lung cancer cells. Constitutive expression of miR-487b completely abolished enhancement of proliferation and invasion of lung cancer cells mediated by overexpression of SUZ12, BMI1, WNT5A, MYC, or KRAS. In contrast, constitutive expression of miR-487b had no effect on proliferation or invasion of lung cancer cells mediated by overexpression of cyclin D1, which is not a predicted target of miR-487b. Collectively, these data suggest that upregulation of each of the 5 aforementioned miR-487b targets contributes to proliferation and invasion potential of lung cancer cells.

Because MYC and KRAS appeared to have more pronounced effects on proliferation and invasion potential of lung cancer 
A Chromosome 14: 101,510,792-101,514,875[+]; intergenic miRNA

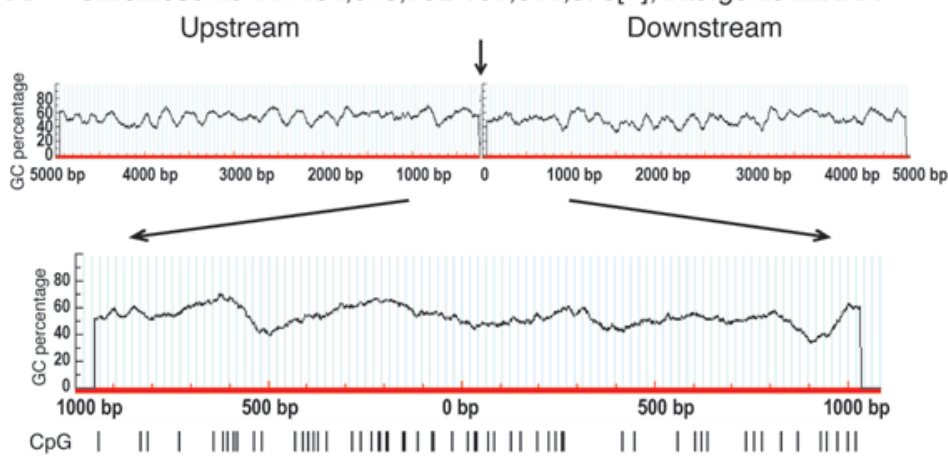

C

$+1(1$ st nucleotide of miR-487b
500 bp $\quad$ genomic locus $14: 101512792)$
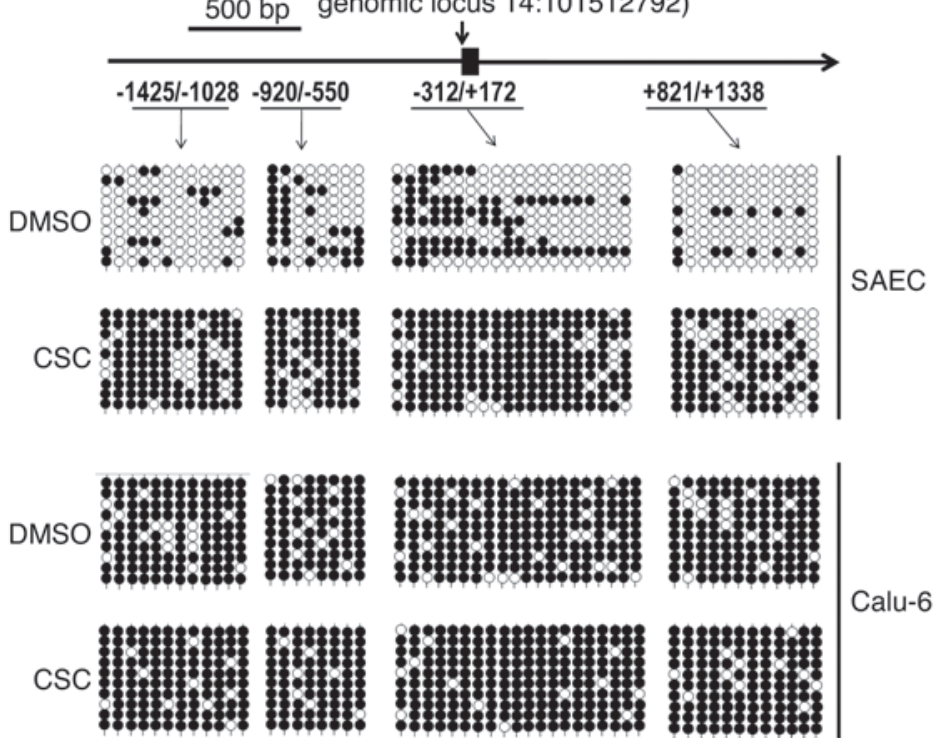

B

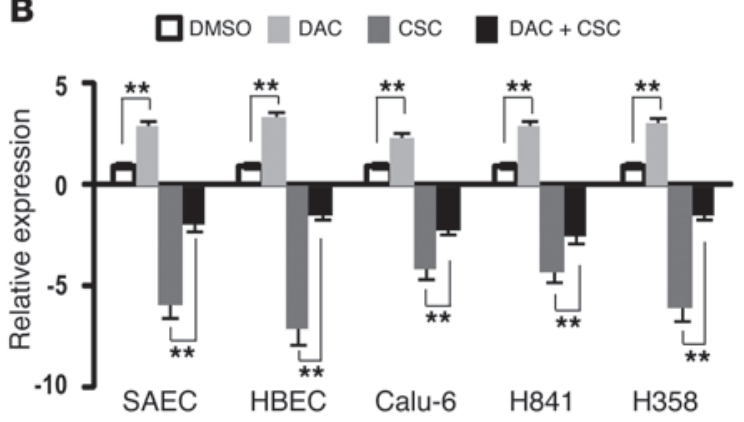

D

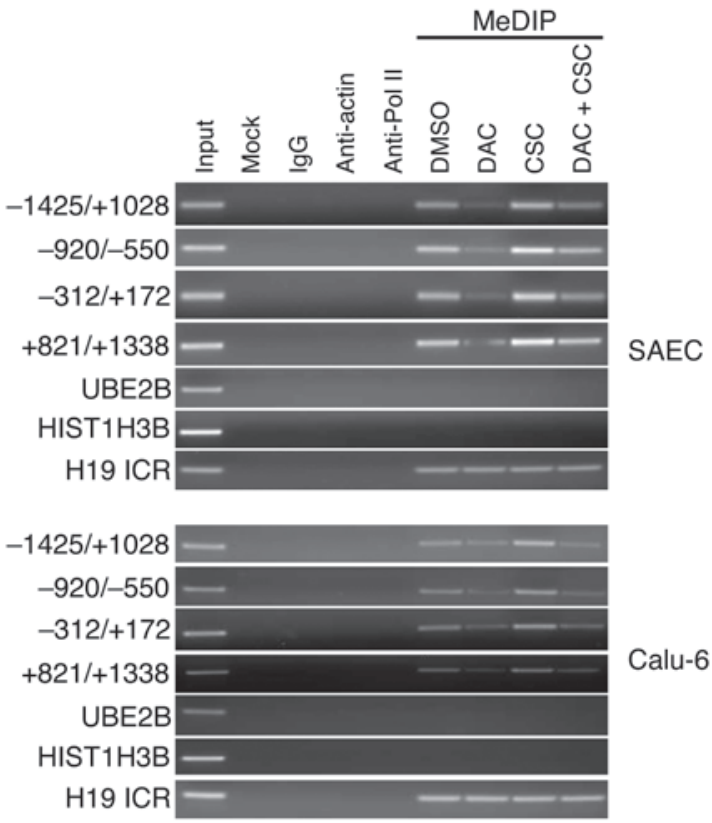

E

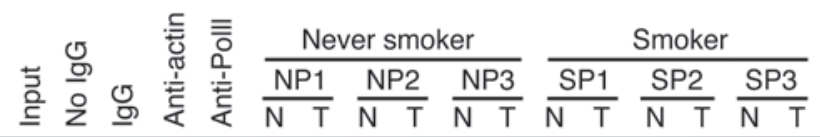

\begin{tabular}{|c|c|c|}
\hline$-1425 /+1028$ & - & $-\quad-\quad---\infty-1-$ \\
\hline$-920 /-550$ & - & $-1+-\infty-\infty-\infty-\infty-\infty$ \\
\hline$-312 /+172$ & $\cdots$ & 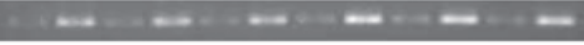 \\
\hline$+821 /+1338$ & $-\infty$ & 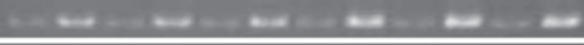 \\
\hline UBE2B & - & \\
\hline HIST1H3B & - & \\
\hline H19 ICR & - & $--m---\cdots---$ \\
\hline
\end{tabular}

\section{Figure 9}

Epigenetic alterations coinciding with CSC-mediated repression of miR-487b. (A) Schematic depiction demonstrating higher CpG density $5 \mathrm{~kb}$ up- and downstream of the miR-487b genomic locus (http://www.urogene.org/methprimer/). (B) qRT-PCR analysis demonstrating that DAC significantly increases expression of primary miR-487b and attenuates CSC-mediated repression of miR-487b in SAECs, HBECs, Calu-6, H841, and $\mathrm{H} 358$ cells. ${ }^{* *} P<0.01$. (C) Bisulfite sequencing of 4 genomic regions around the miR-487b genomic locus, demonstrating that CSC enhances DNA methylation in SAECs. CSC did not alter CpG methylation around miR-487b genomic locus in Calu- 6 cells exhibiting higher pretreatment DNA methylation within these genomic sites. (D) MeDIP analysis of DNA methylation profiles within the miR-487b genomic locus in SAECs and Calu- 6 cells treated with DAC or CSC. DAC significantly decreased methylation near miR-487b in SAECs and Calu- 6 cells and partially abrogated CSC-mediated CpG methylation in these cells. (E) MeDIP analysis of DNA methylation profiles within the miR-487b genomic locus in human lung cancers relative to paired adjacent normal lung tissues. CpG methylation levels near miR-487b were higher in tumors relative to corresponding adjacent normal lung tissues. Furthermore, methylation levels in these regions appeared higher in lung cancers from active/former smokers compared with those from never smokers. 
cells compared with the other 3 miR-487b targets, additional experiments were performed to further examine the impact of these 2 protooncogenes regarding the malignant phenotype of lung cancer cells relative to miR-487b expression. As shown in Figure 6E, combined overexpression of MYC and KRAS did not enhance invasion potential of Calu-6, H841, or H358 lung cancer cells mediated by overexpression of either protooncogene alone. Furthermore, overexpression of miR-487b completely abrogated the enhancement phenomenon mediated by MYC, $K R A S$, or combined expression of these protooncogenes. Deletion of the 3' UTRs in MYC and KRAS abolished the inhibitory effects of miR-487b on MYC- and/or KRAS-mediated invasion potential in these cells. These findings suggest that enhanced invasion of potential of lung cancer cells resulting from repression of miR-487b or overexpression of MYC or KRAS is mediated by redundant mechanisms.

Additional experiments were performed to examine the effects of miR-487b expression on growth of lung cancer cells in vivo. Calu-6, H841, and H358 cells stably transfected with miR-487b or vector controls were inoculated subcutaneously in contralateral flanks of athymic nude mice (10 per group). As shown in Figure 7, $A$ and $B$, volumes and masses of subcutaneous xenografts established from lung cancer cells constitutively expressing miR-487b were significantly smaller than tumors derived from control cells. Subsequent experiments were conducted to examine the effects of miR-487b on lung cancer cells using an orthotopic model. Briefly, nude mice received single transthoracic intrapulmonary injections of Calu- 6 or H358 cells overexpressing miR-487b or respective control cells. Approximately 7 weeks later, mice were euthanized and lungs were evaluated for macroscopic as well as microscopic tumors. Mice inoculated with control Calu- 6 and $\mathrm{H} 358$ cells developed orthotopic tumors with ipsilateral intrapulmonary as well as contralateral metastases. Mice injected with lung cancer cells overexpressing miR-487b had significantly fewer macroscopic as well as microscopic lung nodules and markedly reduced metastases (Figure 7, C and D). Histologic evaluation of lungs from mice injected with control Calu- 6 and H538 cells exhibited numerous pulmonary tumors with invasion into normal lung. This phenomenon was less evident in lungs from mice following orthotopic injection of Calu- 6 and H358 cells constitutively expressing miR-487b (Figure 7E). Consistent with these observations, miR-487b expression significantly decreased the number of contralateral pulmonary metastases in mice inoculated orthotopically with lung cancer cells (Figure 7F).

miR-487b mediates senescence in normal respiratory epithelia and lung cancer cells. Additional experiments were performed to further examine mechanisms by which miR-487b mediates growth arrest in lung cancer cells. Flow cytometry experiments (Figure 8A) demonstrated that overexpression of miR-487b significantly increased, whereas knockdown of miR-487b decreased, $G_{0} / G_{1}$ fraction in normal or immortalized respiratory epithelia (SAECs and HBECs, respectively) as well as lung cancer cells. Annexin V-Cy3 staining experiments (Figure $8 \mathrm{~B}$ ) revealed no change in apoptotic index in lung cancer cells following overexpression or knockdown of miR-487b. Histochemistry experiments demonstrated a marked increase in $\beta$-galactosidase expression in SAECs as well as Calu-6, H841, H358 lung cancer cells following overexpression of miR-487b (Figure 8, C and D). Collectively, these results strongly suggest that miR-487b mediates cell-cycle arrest and senescence in normal respiratory epithelia and lung cancer cells.
Increased CPG methylation coincides with CSC-mediated repression of $m i R-487 b$. A series of experiments was performed to examine mechanisms regulating CSC-mediated repression of miR-487b in lung cancer cells. qPCR techniques were used to examine the region of $14 \mathrm{q} 32$ encoding miR-487b. Because this region is frequently deleted in esophageal carcinomas (25), 2 esophageal cancer lines were used as negative controls, whereas SAECs were used as a positive control for the assay. Copy numbers in lung cancer lines were not appreciably different from those observed in SAECs or HBECs (Supplemental Figure 11A). Subsequent analysis revealed no obvious decrease in DNA copy numbers in 51 lung cancer specimens or paired normal lung tissues (Supplemental Figure 11B). Furthermore, sequencing analysis revealed no alterations in DNA sequence encoding miR-487b in cultured lung cancer lines or primary lung cancer specimens (Supplemental Figure 11C).

Because downregulation of miR-487b in lung cancer cells did not appear attributable to genetic aberrations, additional experiments were performed to determine whether miR-487b repression was mediated by epigenetic mechanisms. CpG density distributed $5 \mathrm{~kb}$ up-and downstream of miR-487b genomic locus (Figure 9A) suggested that DNA methylation may regulate transcription of miR-487b (26). To examine this issue, preliminary experiments were performed to determine whether DNA demethylation induced miR-487b in normal respiratory epithelia and lung cancer cells. qRT-PCR analysis (Figure 9B) revealed that the DNA-demethylating agent, 5-aza-2'-deoxycytidine (DAC) (100 nM $\times 72$ hours), significantly increased expression of primary miR-487b in cultured normal respiratory epithelia and lung cancer cells and markedly attenuated CSC-mediated repression of miR-487b in these cells.

Bisulfite sequencing techniques were used to examine DNA methylation status of 4 genomic regions containing a total of $52 \mathrm{CpG}$ sites around the miR-487b genomic locus. As shown in Figure 9C, DNA methylation levels in these regions were much lower in control SAECs relative to Calu- 6 cells; these observations were consistent with previous data demonstrating lower levels of endogenous miR-487b in cultured lung cancer cells and primary lung cancer specimens relative to cultured normal respiratory epithelia or resected normal lung tissues (Figure 1, A and E). CSC treatment increased CPG methylation around the miR-487b locus in SAECs (Figure 9C); this phenomenon was not apparent in Calu-6 cells, which already exhibited extensive methylation in these regions.

Methylated DNA immunoprecipitation (MeDIP) experiments were performed to further evaluate DNA methylation status of the miR-487b genomic region in SAECs and Calu- 6 cells. Briefly, DNA was immunoprecipitated by anti-methyl CpG antibody, followed by PCR amplification of regions assessed by bisulfite sequencing experiments. As shown in Figure 9D, DAC significantly diminished DNA methylation within the miR-487b genomic locus, as evidenced by decreased PCR products pertaining to DMSO (control) treatment; furthermore, DAC partially abrogated CPG methylation induced by CSC in SAECs and Calu- 6 cells. Similar results were observed following MeDIP analysis of H841, H358, and H1650 lung cancer lines (Supplemental Figure 12). Additional MeDIP experiments demonstrated increased DNA methylation in all 51 lung cancer specimens relative to paired adjacent normal lung tissues (representative results pertaining to 6 paired samples are depicted in Figure 9E; results of the 51 samples are shown in Supplemental Figure 13). MeDIP PCR products appeared to be more pronounced from tobacco-associated lung cancers relative to those from never smokers, although this could not be quantitated precisely. 


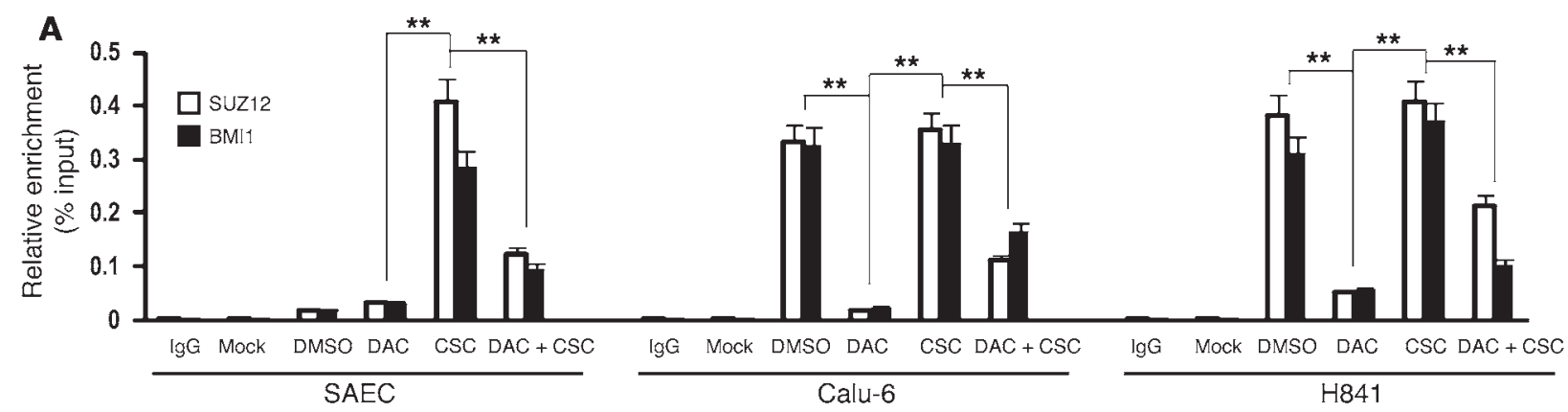

$\mathbf{B}$
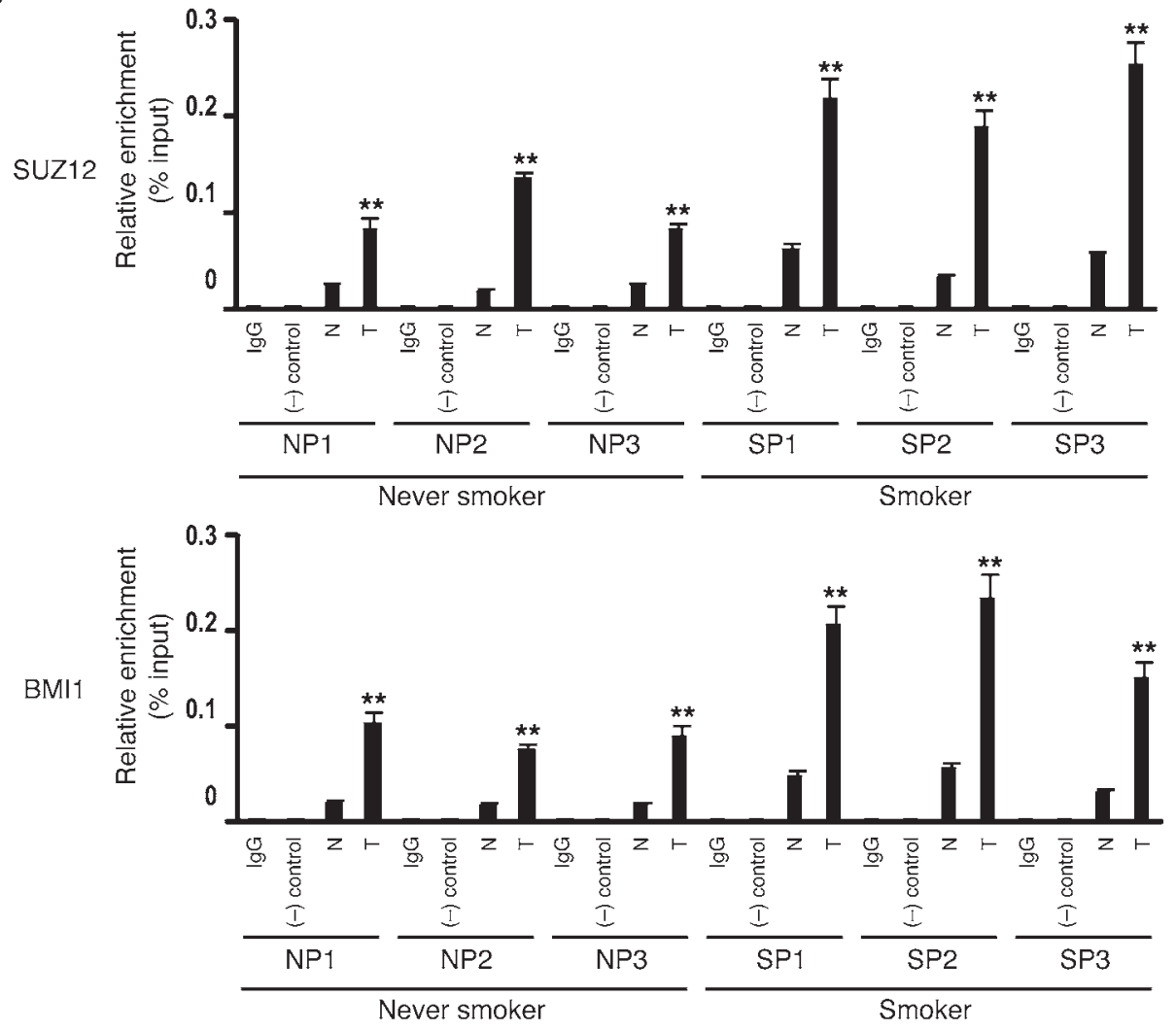

Figure 10

SUZ12 and BMl1 are involved in silencing miR-487b. (A) Quantitative ChIP analysis of SUZ12 and BMI1 levels within the miR-487b genomic locus in SAECs and Calu- 6 and H841 cells. DAC decreased occupancy of SUZ12 and BMI1 in this region in lung cancer cells and significantly attenuated CSC-mediated recruitment of these polycomb proteins to the miR-487b-regulating region. (B) Quantitative ChIP analysis of the miR-487b genomic locus in human lung cancers relative to paired adjacent normal lung tissues. Levels of SUZ12 and BMI1 within the miR-487b genomic locus in tumors $(\mathrm{T})$ were significantly higher than corresponding adjacent normal $(\mathrm{N})$ lung tissues. Furthermore, SUZ12 and BMI1 levels were higher in lung cancers from active/former smokers compared with those from never smokers. $N$ vs. T for each patient: $P<0.01$; never smoker vs. smoker for both $\mathrm{N}$ and $\mathrm{T}: P<0.05 .{ }^{*} P<0.01$.

CSC mediates recruitment of PRC to miR-487. ChIP experiments were performed to determine whether repression of miR-487 by CSC coincided with recruitment of SUZ12 and BMI1 to the miR-487b promoter region. As shown in Figure 10A, control (DMSO-treated) Calu-6 as well as H841 lung cancer cells exhibited increased occupancy of SUZ12 and BMI1 within this region, consistent with increased DNA methylation and downregulation of miR-487b in these cells relative to control SAECs. CSC treatment induced recruitment of SUZ12 and BMI1 to the $\mathrm{miR}-487 \mathrm{~b}$ promoter region in SAECs; this phenomenon was less evident in Calu- 6 cells and H841, which already exhibit relatively high levels of SUZ12 and BMI1 in this region and lower levels of miR-487b expression. DAC treatment had minimal effects on control SAECs, yet markedly decreased levels of SUZ12 and BMI1 within the miR-487b regulatory region in Calu- 6 and H841 cells. Furthermore, DAC treatment significantly attenuated CSC-mediated recruitment of SUZ12 and BMI1 to the miR-487b promoter region in SAECs and Calu6 and H841 cells. Additional quantitative ChIP experiments revealed significantly increased levels of SUZ12 and BMI1 within the miR-487b regulatory region in primary lung cancers relative to paired adjacent normal lung tissues (representative 
A
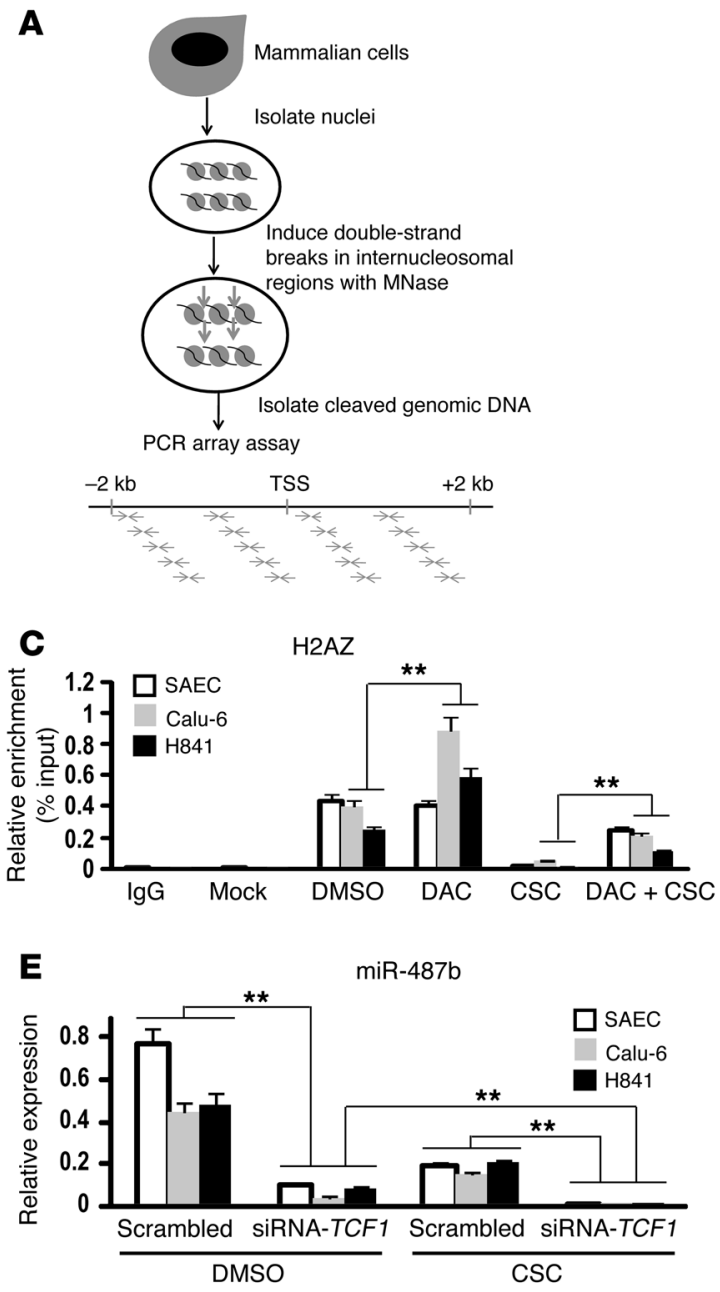

G

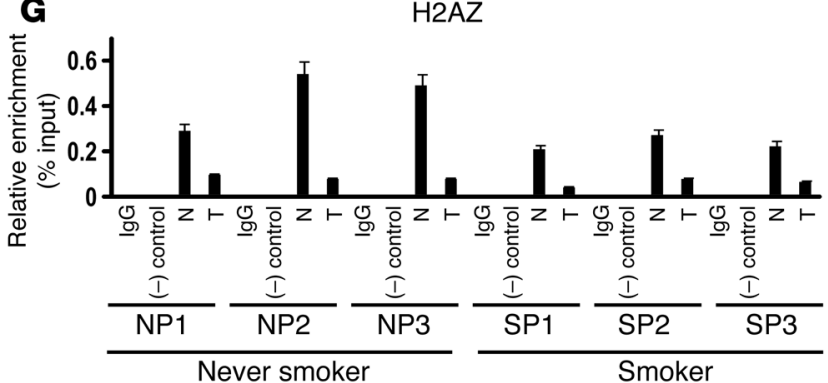

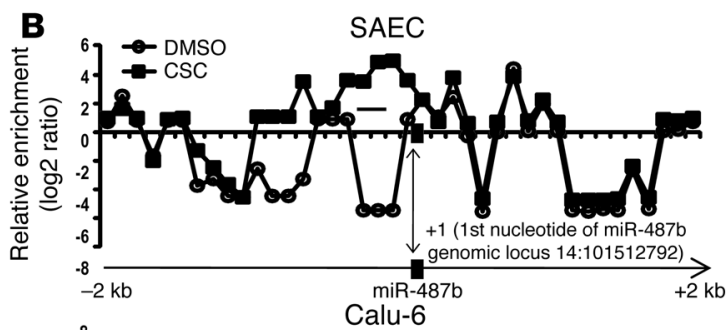
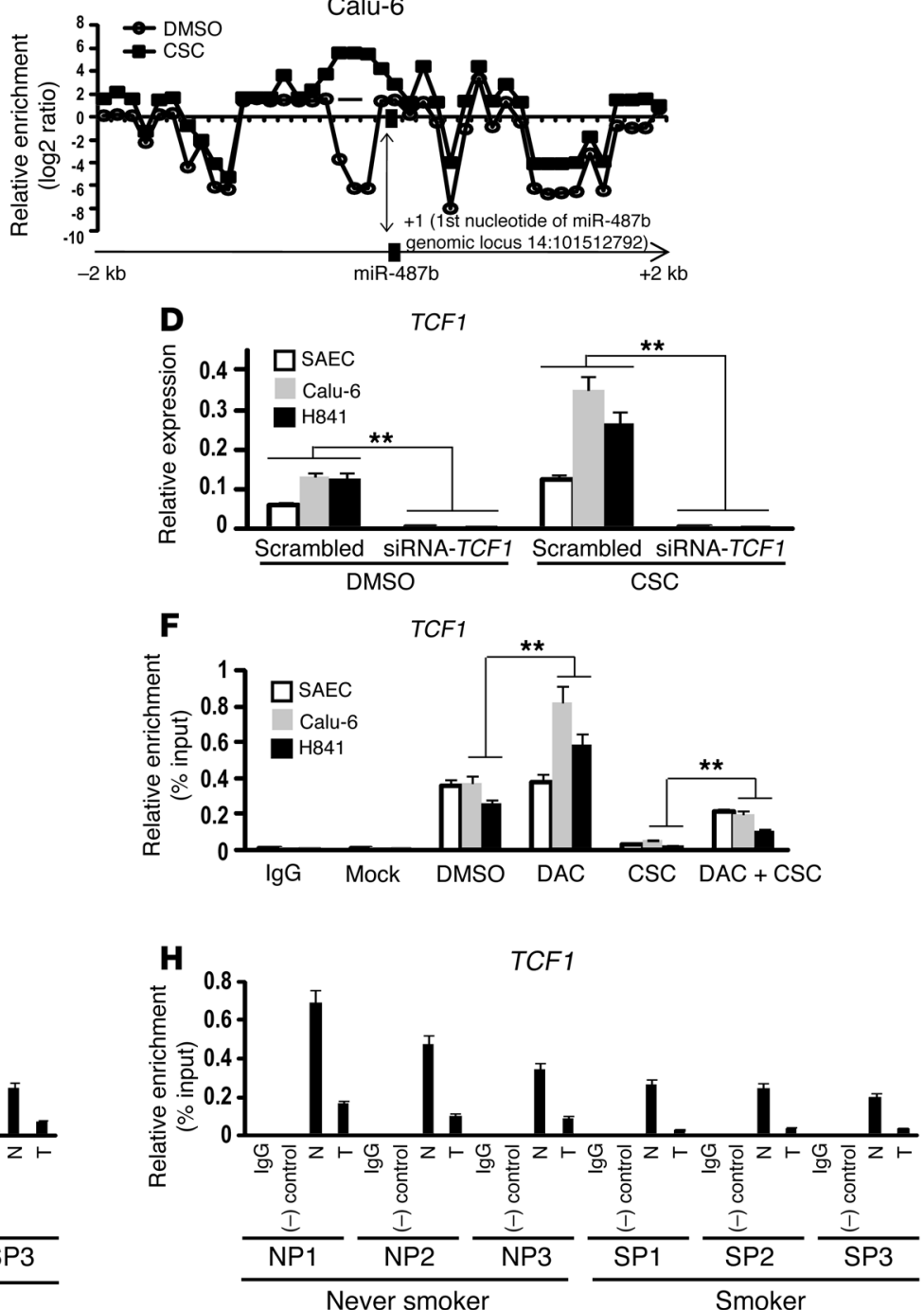

Figure 11

CSC-induced chromatin remodeling coincides with miR-487b transcription. (A) Sketch diagram for MNase protection assay. (B) Results of MNase protection assays depicting 2 upstream NFR $(+50$ to $+350 \mathrm{bp}$ and +650 to $+900 \mathrm{bp})$ of miR-487b in control SAECs and 1 NFR ( +50 to +350$)$ in untreated Calu- 6 cells. CSC induced de novo nucleosome occupancy of these regions. (C) Quantitative ChIP analysis depicting H2AZ levels in the first NFR of miR-487b. DAC partially attenuated CSC-mediated decreases in H2AZ levels. See text for details. (D and E) qRT-PCR analysis demonstrating that CSC induces TCF1 expression in SAECs and Calu-6 and H841 cells (D); knockdown of TCF1 inhibits basal expression of miR-487b in SAECs and Calu-6 and H841 cells and augments CSC-mediated repression of miR-487b in these cells (E). (F) Quantitative ChIP analysis demonstrating decreased occupancy of TCF1 in the first NFR of miR-487b in SAECs and Calu- 6 and H841 cells following CSC exposure. DAC increased TCF1 levels in this NFR in Calu- 6 and H841 cells and attenuated CSC-mediated decreases in TCF1 occupancy in this NFR in SAECs and Calu- 6 and H841 cells. ${ }^{*} P<0.05 ;{ }^{* *} P<0.01$. (G, H) Quantitative ChIP analysis demonstrating significantly lower levels of H2AZ (G) and TCF1 $(\mathbf{H})$ in the first NFR of miR-487b in human lung cancers relative to paired normal lung tissues. H2AZ and TCF1 enrichment levels were lower in lung cancers from active/former smokers compared with those from never smokers. $\mathrm{N}$ vs. T for each patient: $P<0.01$; never smoker vs. smoker for both $\mathrm{N}$ and $\mathrm{T}: P<0.05$. 

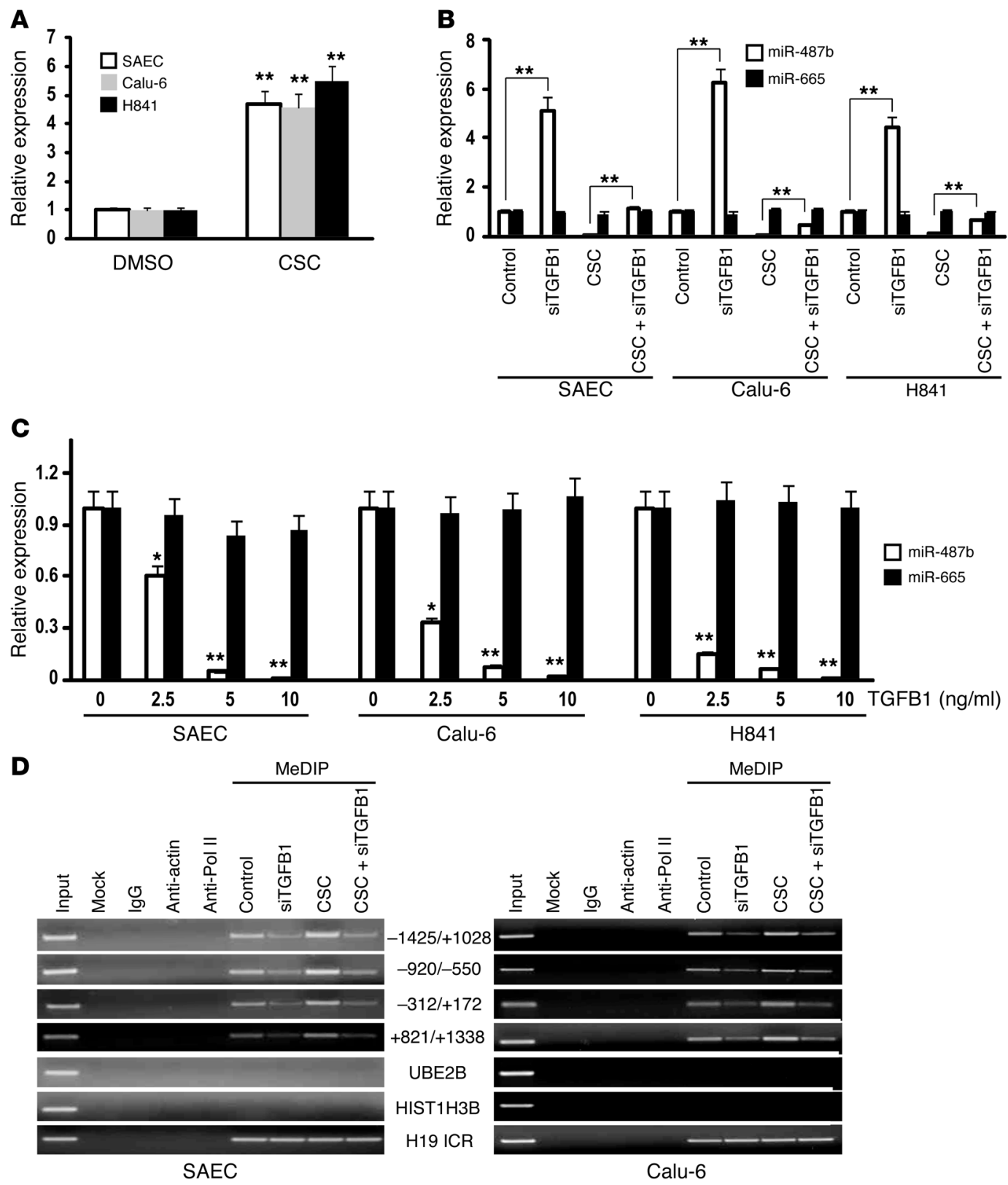

Figure 12

TGFB1 mediates repression of miR-487b by CSC. (A) qRT-PCR analysis demonstrating that CSC enhances TGFB1 expression in SAECs and Calu- 6 and H841 cells. (B) qRT-PCR analysis demonstrating that knockdown of TGFB1 markedly increases miR-487b expression and abrogates CSC-mediated repression of miR-487b in SAECs and to a lesser extent in Calu-6 and H841 cells. Knockdown of TGFB1 had no effect on miR-665 expression in these cells. (C) qRT-PCR analysis demonstrating dose-dependent decreases in miR-487b (but not miR-665) in SAECs and Calu-6 and $\mathrm{H} 841$ cells following 48-hour exposure to recombinant TGFB1. ${ }^{*} P<0.05 ;{ }^{* *} P<0.01$. (D) MeDIP analysis demonstrating that knockdown of TGFB1 markedly decreases DNA methylation levels in untreated SAECs and Calu-6 cells and diminishes CSC-mediated increases in DNA methylation around the miR-487b genomic locus in these cells.

results depicted in Figure 10B). Levels of these polycomb proteins within the miR-487b regulatory region were significantly higher in lung cancers and adjacent normal lung tissues from smokers/former smokers relative to respective specimens from never smokers. Collectively, these findings suggest that recruitment of PRC contributes to CSC-mediated downregulation of $\mathrm{miR}-487 \mathrm{~b}$ in normal respiratory epithelia and stable repression of miR-487b in lung cancer cells.
CSC-induced chromatin remodeling associated with miR-487b transcription. Phased nucleosomal positioning at transcription start sites (TSS) or enhancer elements is associated with active transcription $(27,28)$. As such, additional experiments were performed to determine whether CSC affects nucleosomal positioning around the miR-487b genomic locus in a manner coinciding with repression of this miRNA. Briefly, MNase protection and tiling PCR techniques (Figure 11A and ref. 29) were used to compare mononucleosomes 
prepared from SAECs and Calu- 6 cells cultured in NM with or without CSC for 5 days. As shown in Figure 11B, distinct patterns of MNase sensitivity around the miR-487b genomic locus were observed in CSC-treated SAECs and Calu- 6 cells relative to control cells. Two were identified nucleosome free regions (NFR) (+50 bp to +350 bp and +650 bp to $+900 \mathrm{bp})$ in control SAECs, whereas only $1 \mathrm{NFR}(+50$ to +350$)$ was detected upstream of miR-487b in control Calu- 6 cells. CSC induced de novo nucleosome occupancy of these NFRs in SAECs as well as Calu-6 cells (Figure 11B).

Modulation of H2AZ levels in NFRs of miR-487b by CSC. The histone variant $\mathrm{H} 2 \mathrm{AZ}$ has been linked to gene activation and appears to be confined to small regions flanking TSS $(30,31)$. Incorporation of $\mathrm{H} 2 \mathrm{AZ}$ into chromatin during gene activation depends on availability of NFRs in its target genomic sites $(32,33)$. Quantitative ChIP experiments were performed to determine whether CSC-mediated inhibition of miR-487b in cultured normal respiratory epithelia and lung cancer cells coincided with altered H2AZ levels within the miR-487b promoter region focusing on the common NFR in untreated SAECs and Calu-6 and H841 cells. As shown in Figure 11C, CSC-mediated de novo nucleosome occupancy coincided with decreased H2AZ levels within the first NFR ( +50 bp to +350 bp) of miR-487b in SAECs as well as Calu- 6 and H841 cells. DAC treatment increased H2AZ levels within this NFR in Calu-6 and H841 cells; this phenomenon was not observed in SAECs, which express higher levels of miR-487b and exhibit less induction of miR-487b in response to DAC (Figure 9B). DAC treatment significantly attenuated CSC-mediated decreases in H2AZ levels within this NFR in SAECs as well as Calu-6 and H841 cells (Figure 11C).

Role of TCF1 in CSC-mediated regulation of miR-487b. Additional experiments were performed to further examine mechanisms by which CSC mediates repression of miR-487b. Preliminary software analysis revealed multiple potential binding sites (12 within $1 \mathrm{~Kb}$ upstream of miR-487b) for TCF1, a mediator of canonical ( $\beta$-catenin dependent) Wnt signaling (21). As shown in Figure 11D, qRT-PCR experiments demonstrated that basal levels of TCF1 were somewhat higher in control Calu- 6 as well as H841 lung cancer cells relative to SAECs; CSC exposure enhanced TCF1 expression in these cells. Basal as well as CSC-mediated TCF1 expression was abolished by siRNA targeting TCF1 (Figure 11D). Additional qRTPCR experiments (Figure 11E) revealed that knockdown of TCF1 significantly diminished miR-487b expression in SAECs as well as Calu- 6 and H841 cells and appeared to augment CSC-mediated repression of miR-487b in these cells. Quantitative ChIP experiments (Figure 11F) revealed decreased occupancy of TCF1 in the first NFR ( $+50 \mathrm{bp}$ to $+350 \mathrm{bp})$ of miR-487b in SAECs as well as Calu-6 and H841 cells following CSC exposure. DAC treatment did not appreciably increase TCF1 levels within the first NFR in SAECs, but significantly increased TCF1 levels in this NFR in Calu- 6 and H841 cells. Furthermore, DAC partially abrogated CSC-mediated decreases in TCF1 occupancy within this NFR in SAECs as well as Calu- 6 and H841 cells. Additional ChIP experiments demonstrated reduced levels of $\mathrm{H} 2 \mathrm{AZ}$ and TCF1 within the first NFR of miR-487b in primary lung cancers relative to paired adjacent normal lung tissues; $\mathrm{H} 2 \mathrm{AZ}$ and TCF1 levels in tumors and adjacent normal tissues from active/former smokers were significantly lower than these observed in corresponding samples from never smokers (Figure 11, G and H).

TGFB1 mediates repression of miR-487b by CSC. Recent studies have demonstrated that TGFB1 mediates reversible DNA methylation of miR-200 loci, thereby inducing EMT in Madin-Darby canine kid- ney (MDCK) cells (34). As such, additional experiments were performed to determine whether TGFB1 contributes to CSC-mediated repression of miR-487b. As shown in Figure 12A, qRT-PCR analysis revealed that CSC enhanced TGFB1 expression in SAECs as well as Calu- 6 and H841 cells; siRNA-mediated knockdown of TGFB1 increased miR-487b expression in SAECs as well as Calu-6 and H841 cells and abrogated CSC-mediated repression of miR-487b in SAECs and to a lesser extent in Calu-6 and H841 cells (Figure 12B). Knockdown of TGFB1 had no effects on expression of miR-665, an miRNA that was not modulated by CSC exposure in these cells (Figure 12B). Consistent with these observations, recombinant TGFB1 recapitulated the effects of CSC exposure in a dose-dependent manner in SAECs as well as Calu-6 and H841cells (Figure 12C).

TGFB1-modulates methylation of miR-487b. MeDIP experiments were performed to determine whether TGFB1-mediated repression of miR-487b coincides with increased DNA methylation within the miR-487b regulatory region in normal respiratory epithelia and lung cancer cells. For these experiments, the 4 genomic regions previously evaluated by bisulfite sequencing and MeDIP techniques (Figure 9, C and D) were evaluated. As shown in Figure 12D, decreased PCR products indicative of relative DNA hypomethylation were observed in SAECs as well as Calu- 6 cells following knockdown of TGFB1. As previously shown, CSC increased DNA methylation of miR-487b; knockdown of TGFB1 abrogated the effects of CSC on methylation of the miR-487b regulatory region in SAECs and Calu- 6 cells. For these experiments, $H 19$ imprinting control region (methylated) as well as UBE2B and HIST1H3B (unmethylated) served as controls. Similar results were observed following MeDIP analysis of H841, H358, and H1650 lung cancer lines (Supplemental Figure 14).

\section{Discussion}

Elucidation of the biologic relevance of miRNA alterations during malignant transformation is contingent on identification of respective targets, and delineation of the mechanisms regulating miRNA expression in normal as well as transformed cells. In the present study, we utilized an in vitro model system to gain insight regarding miRNAs potentially contributing to initiation and early progression of tobacco-induced lung cancers. Our analysis revealed that CSC mediated epigenetic repression of miR-487b in normal respiratory epithelia and lung cancer cells. miR-487b specifically targeted SUZ12, BMI1, WNT5A, MYC, and KRAS; overexpression of each of these targets increased proliferation and invasion of lung cancer cells, suggesting that their coordinate upregulation directly enhances the malignant phenotype of these cells. miR-487b expression levels in primary human lung cancer specimens were significantly lower than those observed in adjacent normal lung parenchyma. Repression of miR-487b correlated significantly with overexpression of all $5 \mathrm{miR}-487 \mathrm{~b}$ targets in primary lung cancers relative to normal respiratory epithelia. Downregulation of miR-487b increased proliferation, invasion and tumorigenicity of lung cancer cells, whereas constitutive expression of miR-486b inhibited growth and metastatic potential of lung cancer cells in vitro and in vivo. To the best of our knowledge, these studies are the first to directly implicate epigenetic repression of miR-487b in the pathogenesis of human lung cancers.

Presently, limited information is available regarding miRNA transcription, primarily due to difficulties pertaining to the identification of respective promoter regions, TSS, and other regulatory elements. Approximately 50\% of miRNAs are intragenic, 
with precursors located in exons, introns, or 3' UTRs of protein coding transcripts; these miRNAs share promoters with their respective host genes. However, the remaining 50\% of miRNAs (including miR-487b) are intergenic, with unique TSS located 0.1- to 50-kb upstream from their precursors (reviewed in ref. 35). Several recent studies, utilizing chromatin signatures, cap analysis of gene expression (CAGE) tags, and TSS Seq libraries (35-37) have provided considerable insight regarding potential locations of miRNA promoter regions. However, the vast majority of miRNA promoters identified by software techniques have not been validated; specifically, to date the TSS for miR-487b has not been established. Furthermore, transcription factors and epigenetic alterations coinciding with activation/repression of miRNAs have not been well characterized.

Recent studies indicate that DNA methylation and nucleosomal positioning are mechanistically linked $(38,39)$. Our analysis revealed that miR-487b is embedded in a CpG-rich region, with a functional promoter located approximately 2000 bp upstream of the pre-miR-487b; this site is considerably closer than the TSS predicted by software analysis (49, $414 \mathrm{bp}$ away from the precursor) (35). Furthermore, we observed distinct patterns of nucleosomal positioning within the miR-487b genomic locus in lung cancer cells relative to normal respiratory epithelia. CSC-mediated repression of miR-487b coincided with DNA methylation and altered nucleosome positioning within this region. Consistent with these observations, DAC restored miR-487b expression in lung cancer cells and abrogated CSC-mediated repression of miR-487b in lung cancer as well as normal respiratory epithelial cells. Collectively, these findings suggest that dynamic alterations in chromatin structure are critical determinants of CSC-mediated repression of $\mathrm{miR}-487 \mathrm{~b}$ and provide further evidence that epigenetic repression of tumor suppressor miRNAs contributes to the pathogenesis of human cancers $(1,40)$.

Our analysis revealed that expression of miR-487b is mediated by TCF1. Specifically, basal expression of miR-487b in SAECs and to a lesser extent, Calu- 6 cells coincided with occupancy of TCF1 within the region of the miR-487b TSS; knockdown of TCF1 inhibited miR-487b expression. Interestingly, despite increasing TCF1 expression, CSC decreased occupancy of TCF1 within the miR-487b promoter region, a phenomenon that was abrogated by DAC. These findings indicate that basal expression of miR-487b in normal respiratory epithelia is mediated, at least in part, by canonical Wnt signaling and suggest a potential feedback mechanism by which miR-487b regulates Wnt-mediated proliferation in normal respiratory epithelia. Formation of heterochromatin inhibits access of TCF1 to recognition elements within the miR-487b region, thereby silencing this miRNA during tobacco-induced pulmonary carcinogenesis. These findings are analogous to recent observations demonstrating that chromatin structure directly affects the ability of Sp1 and Sp3 to activate TGFB1 (41).

Data presented in this manuscript extend our recent observations that CSC activates miR-31, targeting several Wnt antagonists in lung cancer cells and normal respiratory epithelia (10). Although our present experiments have not directly demonstrated that epigenetic repression of miR-487b by cigarette smoke enhances pluripotency of lung cancer cells, it is intriguing that all $5 \mathrm{miR}$ $487 \mathrm{~b}$ targets mediate cancer stem cell phenotypes (16, 21, 42) and are potentially relevant regarding prognosis in lung cancer patients. SUZ12 and BMI1 are core components of PRC that are critical determinants of more than 1,000 genes in stem cells (43).
BMI1 is required for expansion of bronchioalveolar stem cells, the putative cells of origin of pulmonary adenocarcinomas (44), and stemness signatures mediated in part by BMI1 and SUZ12 in lung cancers correlate with aggressive tumor phenotypes and decreased survival of patients with these malignancies (45). WNT5A activates planar cell polarity pathways during normal organogenesis and cancer dissemination $(22,46)$. Overexpression of WNT5A increases proliferation, migration, invasion, and tumorigenicity of lung cancer cells in part via modulation of HOX family genes (D. Schrump et al., unpublished observations); elevated intratumoral levels of WNT5A correlate with increased neoangiogenesis, dissemination of disease, and decreased survival of lung cancer patients (47). MYC and KRAS activate a variety of pathways facilitating initiation and progression of lung cancer $(24,48)$, and overexpression of these oncogenes correlates with treatment resistance and poor survival in lung cancer patients $(49,50)$. Collectively, these observations strongly suggest that epigenetic repression of miR-487b enhances stemness phenotype of lung cancers and are consistent with our recent studies demonstrating that cigarette smoke upregulates $A B C G 2$, increases pluripotent side population, and activates a variety of stem cell signaling pathways in lung cancer cells (51). Additional studies using gene expression profiling and flow cytometry techniques are in progress to more comprehensively examine the effects of miR-487b on global gene expression and stem cell phenotype of lung cancer cells and to more precisely define the constituents of cigarette smoke that induce stemness during pulmonary carcinogenesis.

TGFB1 exhibits highly pleiotropic effects during normal cellular homeostasis and organogenesis; aberrant TGFB1 signaling has been implicated in abnormal remodeling of small airways in chronic smokers (52) as well as progression of human lung cancers (53-55). Recent studies have demonstrated that TGF- $\beta$ signaling modulates and can be regulated by miRNA expression via complex regulatory networks (56). Although several investigators observed no upregulation of TGFB1 in cultured HBECs following brief exposures ( $<24$ hours) to cigarette smoke (57) or brushings from proximal airways in smokers (58), our experiments clearly showed that 5-day CSC exposure increased TGFB1 expression in cultured normal respiratory epithelia and lung cancer cells. Overexpression of TGFB1 or exogenous recombinant TGFB1 downregulated miR-487b in SAECs and Calu- 6 cells. Knockdown of TGFB1 abrogated CSC-mediated repression of miR-487b. These data suggest that the effects of CSC on miR-487b expression are mediated to a significant extent by TGFB1 and are consistent with recent studies demonstrating that this cytokine induces DNA methylation-mediated silencing of miR-200 (34). Whereas the specific DNMTs and signaling pathways, which mediate CSC-induced methylation of miR-487b in respiratory epithelia, are a focus of ongoing experiments, it is noteworthy that several recent studies have demonstrated that inhibition of TGF- $\beta$-smad signaling decreases expression and DNA binding activity of DNMT1, resulting in demethylation of several genes including TGFB receptors in prostate cancer cells (59) as well as CDH1, CGN, CLD4, and KLK10 in breast cancer cells (60). Conceivably similar mechanisms contribute to epigenetic repression of miR-487b by cigarette smoke.

Our studies not only provide insight regarding the pathogenesis of human lung cancers, but also suggest a possible mechanism by which smoking status at diagnosis or during treatment affects survival of lung cancer patients $(61,62)$. Furthermore, our studies highlight the potential for epigenetic interventions to reverse 
tobacco-induced reprogramming in lung cancer cells. Under exposure conditions approximating those achieved in clinical settings (63), DAC restored miR-487b expression in lung cancer cells. Collectively, our studies warrant further analysis of epigenetic mechanisms regulating miRNA expression during human pulmonary carcinogenesis and the utilization of chromatin remodeling agents to restore miR-487b expression for lung cancer therapy.

\section{Methods}

Cell lines and treatment conditions. All lung cancer lines were obtained from ATCC. NCI-SB-EsC2 (EsC2) was established in our lab; OE-33 was purchased from Sigma-Aldrich. All cancer lines were maintained in RPMI medium supplemented with $10 \%$ FCS, $10 \mathrm{mM}$ glutamic acid, and $1 \%$ penicillin/streptomycin (NM). Primary normal human SAECs were obtained from Lonza Inc. and cultured per vendor instructions. Immortalized HBECs were provided by John D. Minna (UT Southwestern, Dallas, Texas, USA) and cultured as described (64). CSCs derived from Kentucky Reference $3 \mathrm{R} 4 \mathrm{~F}$ research blend cigarettes (University of Kentucky) were prepared as described (64) and resuspended at a concentration of $10 \mathrm{mg} \mathrm{tar} / \mathrm{ml}$ in DMSO as stock concentration. For smoke-exposure experiments, cells were cultured in 10-cm dishes in appropriate NM with DMSO or NM with CSC $(0.025 \mathrm{mg} / \mathrm{ml})$. Medium was changed daily with the addition of fresh CSC or DMSO control. Cells were subcultured as necessary and harvested at various time points for analysis. For recombinant TGFB1 protein experiments, cells were cultured in 10-cm dishes in appropriate NM with or without TGFB1 (Santa Cruz Biotechnology Inc.) for 48 hours followed by analysis.

Human tissues. Primary lung cancer specimens and adjacent histologically normal lung parenchyma were harvested intraoperatively from patients undergoing potentially curative resections. All tissues were immediately snap-frozen and stored for further analysis. A portion of harvested tissue was sent for immediate histologic confirmation by an independent, anatomic pathologist in a blinded manner. The small RNA fraction was isolated with the RT2 qPCR-Grade miRNA Isolation kit (QIAGEN), and miRNA expression was quantified with the qRT-PCR miRNA Detection Kit (ABI). miRNA overexpression and inbibition. Precursor and inhibitor miRNAs, pMiRH487bPA-1 and PCDH-CMV-MCS-EF1-copGFP negative control, were purchased from SBI (System Biosciences). Anti-miR-487b oligos and a scramble negative oligo control were purchased from ABI. miRNA precursors or antisense oligos (50 $\mathrm{nM}$ or $10 \mathrm{nM}$ respectively) were transfected into cells using Lipofectamine 2000 (Invitrogen), followed by analysis 48-72 hours later.

Reporter vectors and DNA constructs. The 3' UTRs of WNT5A (4377 bp), BMI1 (1946 bp), and SUZ12 (2027 bp) were PCR amplified from human SAEC cDNA, and inserted downstream of CMV-driven firefly luciferase cassette in the PMIR-REPORT vector (Ambion) between HindIII and SpeI sites. Mutant reporter plasmids (3' UTRs of WNT5A, BMI1, and SUZ12) were created by using QuikChange Site-Directed Mutagenesis Kit (Stratagene). All insert fragments were confirmed by direct sequencing.

Luciferase miRNA target reporter assays. For miRNA target validation, approximately $2 \times 10^{4}$ SAECs per well in 24-well plates were transiently transfected with 25 to $50 \mathrm{ng}$ of each of $3^{\prime}$ UTR-pMIR-REPORT constructs or those mutated constructs (Ambion), and 30 pmol of pre-miR-487b (SBI). Renilla luciferase vector was used to normalize transfection efficiency. Approximately 24 hours after transfection, firefly and Renilla luciferase activities were assayed. Normalized relative light units represent firefly luciferase activity/Renilla luciferase activity.

Proliferation assays. Cells were plated at a concentration of $5 \times 10^{4}$ cells per well in 24-well plates and cultured in NM with or without plasmid transfection or anti-miR-487b oligos. Triplicate wells were harvested and counted by trypan blue exclusion techniques.

qRT-PCR. See Supplemental Methods.
ChIP. Cells were crosslinked with $1 \%$ formaldehyde, lysed, and sonicated on ice to generate DNA fragments with an average length of $200-800$ bp. After preclearing, $1 \%$ of each sample was saved as input fraction. Immunoprecipitation was performed using ChIP-grade antibodies specifically recognizing TCF1, SUZ12, BMI1, and H2AZ (Abcam), RNA polymerase II (Upstate), or IgG control (Supplemental Table 2). DNA was eluted and purified from complexes, followed by PCR amplification of the target promoters or genomic loci using primers listed in Supplemental Table 1 and conditions as described (65).

siRNA and shRNA knockdown. See Supplemental Methods.

Western blot analysis. See Supplemental Methods.

RT-PCR arrays. See Supplemental Methods.

miRNA PCR array analysis. See Supplemental Methods. Array data have been deposited at the Gene Expression Omnibus (GEO GSE42962).

Ago CLIP. Cells were harvested, and CLIP experiments were performed using either anti-AGO family or elF2C antibodies as described (10). Briefly, cells were crosslinked with irradiation for $400 \mathrm{~mJ} / \mathrm{cm}^{2}$ and additional $200 \mathrm{~mJ} / \mathrm{cm}^{2}$ in Stratalinker, and lysed to generate RNA/protein (AGO) complexes $(10,66)$. After preclearing, $1 \%$ of each sample was saved as input fraction. Immunoprecipitation was performed using specific antibodies against either the AGO family (Millipore), elF2C (Santa Cruz Biotechnology Inc.), or control IgG. RNA was isolated and purified from complexes, followed by PCR amplification of 3' UTRs of miR-487b targets.

MeDIP assay. Genomic DNA was sonicated to produce fragments ranging in size from 300 to $1000 \mathrm{bp}$. Five micrograms of fragmented DNA was used for a standard MeDIP assay $(29,67)$ and precipitated with $10 \mu \mathrm{l}$ monoclonal antibody against 5-methylcytidine (Eurogentec) followed by incubation with protein A agarose beads. DNA was recovered by phenolchloroform extraction followed by ethanol precipitation. Primers for PCR (listed in Supplemental Table 1) were chosen within 1000-bp up- and downstream of the transcription start site of miR-487b; amplicon sizes varied between 200 and $350 \mathrm{bp}$.

Bisulfite sequencing. Genomic DNA was subjected to bisulfite treatment using QIAGEN Epifect kit (QIAGEN). The PCR products were separated in agarose gels, purified, and subcloned as described $(65,67)$. Primers for bisulfite sequencing are listed in Supplemental Table 1.

MNase protection analysis. The assay was performed as described previously (68). Briefly, after lysis of single-cell suspensions, chromatin was digested with MNase (Thermo Scientific); DNA was extracted by phenol:chloroform and separated on a $1.5 \%$ agarose gel. This procedure yielded approximately $80 \%$ DNA from mononucleosomes ( $150 \mathrm{bp}$ ) and approximately $20 \%$ DNA from di- and trinucleosomes ( $\sim 300$ and $\sim 450 \mathrm{bp}$, respectively). The mononucleosomal DNA was excised, gel purified, and used as a PCR template.

In vitro invasion. Cell invasion was evaluated in vitro using ECM-coated semipermeable modified Boyden chambers (Millipore) as previously described (69). Full details are available in Supplemental Methods.

Murine xenograft experiments. Calu- 6 and H358 cells transfected with pCDH-CMV-MCS-EF1-copGFP vector control and PMiR-H487bPA-1 were suspended in PBS at a concentration of $1 \times 10^{6}$ cells $/ 100 \mu \mathrm{l}$ and inoculated subcutaneously into contralateral flanks of athymic nude mice (10 mice per treatment group per experiment). Mice were monitored twice weekly, and tumor volumes were calculated on the basis of perpendicular diameters. Approximately 25 days and 35 days later for Calu- 6 and H358 experiments, respectively, mice were euthanized and evaluated for percentage of tumor take and mass of excised xenografts. Thereafter, tumor tissues were snap frozen in liquid nitrogen and stored for future analysis.

Orthotopic intralung metastasis model. $2 \times 10^{5}$ cells from Calu- 6 or $\mathrm{H} 358$ cells stably expressing miR-487b or control vectors were injected into the right hemithoracic of nude mice. Seven weeks after injection of tumor cells, mice were euthanized and lungs were excised for histopathological evaluation. 
Macroscopic tumor nodes were recorded following formaldehyde fixation of lung blocks for 48 hours. Gross number of lung tumor nodes was counted based on pathological examination of each sectioned lobe. At least one paraffin-embedded section slide from each lobe per mouse was prepared for $\mathrm{H} \& \mathrm{E}$ staining followed by image recording and measurement of total microscopic invasive nodules per mouse.

Cell cycle, senescence, and apoptosis assays. See Supplemental Methods.

Statistics. Data are presented as means \pm SEM. Differences between matched tumor and normal tissues from lung cancer patients were evaluated with the signed-rank test. Groups were compared with the Wilcoxon-Mann-Whitney test. Confidence intervals for the differences in medians between 2 groups were computed by the bias-corrected and accelerated bootstrapping methods. Differences in tumor volumes between opposing flanks of tumor-bearing mice were tested with the Wilcoxon signed-rank test at 5-day intervals. Confidence intervals for the median of flank differences were computed as exact nonparametric 2-tailed confidence intervals based on the Wilcoxon signed-rank test. $P$ values were adjusted for multiple testing by complete resampling. $P<0.05$ was considered significant.

Study approval. Primary lung cancer specimens and adjacent histologically normal lung parenchyma were harvested intraoperatively from patients undergoing potentially curative resections at the NIH Clinical Center
(Bethesda, Maryland, USA) according to NIH internal review boardapproved protocols requiring written informed consent. All tissue specimens were bar coded and stored in the Thoracic Oncology Laboratory, Surgery Branch, NCI. All animal procedures were approved by the National Cancer Institute Animal Care and Use Committee and were in accordance with the NIH Guide for the Care and Use of Laboratory Animals.

\section{Acknowledgments}

The authors express their gratitude to Jan Pappas for assistance regarding manuscript preparation. This work was supported by NCI Intramural grants ZIA BC 011122 (to D.S. Schrump) and ZIA BC 011418 (to D.S. Schrump) as well as the Steven J. Solarz Memorial Fund.

Received for publication October 4, 2011, and accepted in revised form January 3, 2013.

Address correspondence to: David S. Schrump, Thoracic Oncology Section, Surgery Branch, Center for Cancer Research, NCI, 10 Center Drive; Room 4-3942, Bethesda, Maryland 20892, USA. Phone: 301.496.2128; Fax: 301.451.6934; E-mail: David_ Schrump@nih.gov.
1. Malumbres M. miRNAs and cancer: an epigenetics view [published online ahead of print July 4, 2012]. Mol Aspects Med. doi:10.1016/j.mam.2012.06.005.

2. Jansson MD, Lund AH. MicroRNA and cancer. $\mathrm{Mol}$ Oncol. 2012;6(6):590-610.

3. Iorio MV, Croce CM. MicroRNA dysregulation in cancer: diagnostics, monitoring and therapeutics. A comprehensive review. EMBO Mol Med. 2012; 4(3):143-159.

4. Lee D, Shin C. MicroRNA-target interactions: new insights from genome-wide approaches. Ann N Y Acad Sci. 2012;1271:118-128.

5. Zardo G, et al. Transcriptional targeting by microRNA-Polycomb complexes: A novel route in cell fate determination. Cell Cycle. 2012;11(19):3543-3549.

6. Toscano-Garibay JD, Aquino-Jarquin G. Regulation exerted by miRNAs in the promoter and UTR sequences: MDR1/P-gp expression as a particular case. DNA Cell Biol. 2012;31(8):1358-1364.

7. Markou A, Liang Y, Lianidou E. Prognostic, therapeutic and diagnostic potential of microRNAs in non-small cell lung cancer. Clin Chem Lab Med. 2011; 49(10):1591-1603

8. Enfield KS, Pikor LA, Martinez VD, Lam WL. Mechanistic roles of noncoding RNAs in lung cancer biology and their clinical implications. Genet Res Int. 2012;2012:737416

9. Thun MJ, DeLancey JO, Center MM, Jemal A, Ward EM. The global burden of cancer: priorities for prevention. Carcinogenesis. 2010;31(1):100-110.

10. Xi S, et al. Cigarette smoke induces C/EBP-betamediated activation of miR-31 in normal human respiratory epithelia and lung cancer cells. PLoS One. 2010;5(10):e13764

11. Liu X, et al. MicroRNA-31 functions as an oncogenic microRNA in mouse and human lung cancer cells by repressing specific tumor suppressors. J Clin Invest. 2010;120(4):1298-1309.

12. Liu B, et al. MiR-26a enhances metastasis potential of lung cancer cells via AKT pathway by targeting PTEN. Biochim Biophys Acta. 2012;1822(11):1692-1704.

13. Tellez CS, et al. EMT and stem cell-like properties associated with miR-205 and miR-200 epigenetic silencing are early manifestations during carcinogen-induced transformation of human lung epithelial cells. Cancer Res. 2011;71(8):3087-3097.

14. Ahn YH, et al. ZEB1 drives prometastatic actin cytoskeletal remodeling by downregulating miR-34a expression. J Clin Invest. 2012;122(9):3170-3183.

15. Huo JS, Zambidis ET. Pivots of pluripotency: The roles of non-coding RNA in regulating embryonic and induced pluripotent stem cells. Biochim Biophys Acta. 2013;1830(2):2385-2394.

16. Han JW, Yoon YS. Epigenetic landscape of pluripotent stem cells. Antioxid Redox Signal. 2012; 17(2):205-223.

17. Boeri M, Pastorino U, Sozzi G. Role of microRNAs in lung cancer: microRNA signatures in cancer prognosis. Cancer J. 2012;18(3):268-274.

18. Gattolliat CH, et al. Expression of miR-487b and miR-410 encoded by $14 \mathrm{q} 32.31$ locus is a prognostic marker in neuroblastoma. Br J Cancer. 2011; 105(9):1352-1361.

19. Christophersen NS, Helin K. Epigenetic control of embryonic stem cell fate. J Exp Med. 2010; 207(11):2287-2295.

20. Sawarkar R, Paro R. Interpretation of developmental signaling at chromatin: the Polycomb perspective. Dev Cell. 2010;19(5):651-661.

21. Espada J, Calvo MB, Diaz-Prado S, Medina V. Wnt signalling and cancer stem cells. Clin Transl Oncol. 2009;11(7):411-427.

22. Konigshoff M, Eickelberg O. WNT signaling in lung disease: a failure or a regeneration signal? $\mathrm{Am}$ J Respir Cell Mol Biol. 2010;42(1):21-31.

23. Hussain M, et al. Tobacco smoke induces polycomb-mediated repression of Dickkopf-1 in lung cancer cells. Cancer Res. 2009;69(8):3570-3578.

24. Tran PT, et al. Combined inactivation of MYC and $\mathrm{K}-\mathrm{RAS}$ oncogenes reverses tumorigenesis in lung adenocarcinomas and lymphomas. PLoS One. 2008; 3(5):e2125.

25. Ihara $Y$, et al. Allelic imbalance of $14 \mathrm{q} 32$ in esophageal carcinoma. Cancer Genet Cytogenet. 2002; 135(2):177-181.

26. Cheung $\mathrm{HH}$, et al. Methylation of an intronic region regulates miR-199a in testicular tumor malignancy. Oncogene. 2011;30(31):3404-3415.

27. Tsankov AM, Thompson DA, Socha A, Regev A, Rando OJ. The role of nucleosome positioning in the evolution of gene regulation. PLoS Biol. 2010;8(7):e1000414.

28. Bai L, Morozov AV. Gene regulation by nucleosome positioning. Trends Genet. 2010;26(11):476-483.

29. Tao Y, et al. Lsh, chromatin remodeling family member, modulates genome-wide cytosine meth- ylation patterns at nonrepeat sequences. Proc Natl Acad Sci U S A. 2011;108(14):5626-5631.

30. Barski A, et al. High-resolution profiling of histone methylations in the human genome. Cell. 2007;129(4):823-837.

31. Kotova E, Lodhi N, Jarnik M, Pinnola AD, Ji Y, Tulin AV. Drosophila histone $\mathrm{H} 2 \mathrm{~A}$ variant $(\mathrm{H} 2 \mathrm{Av})$ controls poly(ADP-ribose) polymerase 1 (PARP1) activation in chromatin. Proc Natl Acad Sci U S A. 2011; 108(15):6205-6210.

32. Creyghton MP, et al. H2AZ is enriched at polycomb complex target genes in ES cells and is necessary for lineage commitment. Cell. 2008;135(4):649-661.

33. Millar CB, Xu F, Zhang K, Grunstein M. Acetylation of H2AZ Lys 14 is associated with genome-wide gene activity in yeast. Genes Dev. 2006;20(6):711-722.

34. Gregory PA, et al. An autocrine TGF-beta/ZEB/ miR-200 signaling network regulates establishment and maintenance of epithelial-mesenchymal transition. Mol Biol Cell. 2011;22(10):1686-1698.

35. Chien $\mathrm{CH}$, et al. Identifying transcriptional start sites of human microRNAs based on high-throughput sequencing data. Nucleic Acids Res. 2011; 39(21):9345-9356.

36. Ozsolak F, et al. Chromatin structure analyses identify miRNA promoters. Genes Dev. 2008; 22(22):3172-3183.

37. Zhu S, Jiang Q, Wang G, Liu B, Teng M, Wang Y. Chromatin structure characteristics of pre-miRNA genomic sequences. BMC. Genomics. 2011;12:329.

38. Chodavarapu RK, et al. Relationship between nucleosome positioning and DNA methylation. Nature. 2010;466(7304):388-392.

39. Kelly TK, Liu Y, Lay FD, Liang G, Berman BP, Jones PA. Genome-wide mapping of nucleosome positioning and DNA methylation within individual DNA molecules. Genome Res. 2012;22(12):2497-2506.

40. Wong KY, Yu L, Chim CS. DNA methylation of tumor suppressor miRNA genes: a lesson from the miR-34 family. Epigenomics. 2011;3(1):83-92.

41. Lee JJ, et al. Accessible chromatin structure permits factors Sp1 and Sp3 to regulate human TGFBI gene expression. Biochem Biophys Res Commun. 2011; 409(2):222-228.

42. Motohara T, et al. Transient depletion of p53 followed by transduction of c-Myc and K-RAS converts ovarian stem-like cells into tumor-initiating cells. Carcinogenesis. 2011;32(11):1597-1606. 
43. Easwaran $\mathrm{H}$, et al. A DNA hypermethylation module for the stem/progenitor cell signature of cancer. Genome Res. 2012;22(5):837-849.

44. Dovey JS, Zacharek SJ, Kim CF, Lees JA. Bmi1 is critical for lung tumorigenesis and bronchioalveolar stem cell expansion. Proc Natl Acad Sci U S A. 2008; 105(33):11857-11862.

45. Glinsky GV. "Stemness” genomics law governs clinical behavior of human cancer: implications for decision making in disease management. J Clin Oncol. 2008;26(17):2846-2853.

46. Kikuchi A, Yamamoto H, Sato A, Matsumoto S. WNT5A: its signalling, functions and implication in diseases. Acta Physiol (Oxf). 2012;204(1):17-33.

47. Huang CL, et al. WNT5A expression is associated with the tumor proliferation and the stromal vascular endothelial growth factor--an expression in non-small-cell lung cancer. J Clin Oncol. 2005; 23(34):8765-8773.

48. Govindan R, et al. Genomic landscape of non-small cell lung cancer in smokers and never-smokers. Cell. 2012;150(6):1121-1134.

49. Allen TD, Zhu CQ, Jones KD, Yanagawa N, Tsao MS, Bishop JM. Interaction between MYC and MCL1 in the genesis and outcome of non-smallcell lung cancer. Cancer Res. 2011;71(6):2212-2221.

50. Levallet G, et al. High TUBB3 expression, an independent prognostic marker in patients with early non-small cell lung cancer treated by preoperative chemotherapy, is regulated by K-RAS signaling pathway. Mol Cancer Ther. 2012;11(5):1203-1213.

51. Zhang M, et al. Mithramycin represses basal and cigarette smoke-induced expression of ABCG2 and inhibits stem cell signaling in lung and esophageal cancer cells. Cancer Res. 2012;72(16):4178-4192.
52. Konigshoff M, Kneidinger N, Eickelberg O. TGFbeta signaling in COPD: deciphering genetic and cellular susceptibilities for future therapeutic regimen. Swiss Med Wkly. 2009;139(39-40):554-563.

53. Zhang HJ, et al. Transforming growth factor-beta 1 promotes lung adenocarcinoma invasion and metastasis by epithelial-to-mesenchymal transition. Mol Cell Biochem. 2011;355(1-2):309-314.

54. Hasegawa Y, Takanashi S, Kanehira Y, Tsushima T, Imai T, Okumura K. Transforming growth factorbeta1 level correlates with angiogenesis, tumor progression, and prognosis in patients with nonsmall cell lung carcinoma. Cancer. 2001;91(5):964-971.

55. Zhao L, et al. Changes of circulating transforming growth factor-beta 1 level during radiation therapy are correlated with the prognosis of locally advanced non-small cell lung cancer.J Thorac Oncol. 2010;5(4):521-525

56. Butz H, Racz K, Hunyady L, Patocs A. Crosstalk between TGF-beta signaling and the microRNA machinery. Trends Pharmacol Sci. 2012;33(7):382-393.

57. Spira A, et al. Effects of cigarette smoke on the human airway epithelial cell transcriptome. Proc Natl Acad Sci U S A. 2004;101(27):10143-10148.

58. Maunders H, Patwardhan S, Phillips J, Clack A, Richter A. Human bronchial epithelial cell transcriptome: gene expression changes following acute exposure to whole cigarette smoke in vitro. Am J Physiol Lung Cell Mol Physiol. 2007; 292(5):L1248-L1256.

59. Zhang Q, et al. TGF-beta regulates DNA methyltransferase expression in prostate cancer, correlates with aggressive capabilities, and predicts disease recurrence. PLoS One. 2011;6(9):e25168.

60. Papageorgis $P$, et al. Smad signaling is required to maintain epigenetic silencing during breast cancer progression. Cancer Res. 2010;70(3):968-978.

61. Janjigian YY, et al. Pack-years of cigarette smoking as a prognostic factor in patients with stage IIIB/IV nonsmall cell lung cancer. Cancer. 2010;116(3):670-675.

62. Waller LL, Weaver KE, Petty WJ, Miller AA. Effects of continued tobacco use during treatment of lung cancer. Expert Rev Anticancer Ther. 2010; 10(10):1569-1575.

63. Schrump DS, et al. Phase I study of decitabinemediated gene expression in patients with cancers involving the lungs, esophagus, or pleura. Clin Cancer Res. 2006;12(19):5777-5785.

64. Liu F, et al. Epigenomic alterations and gene expression profiles in respiratory epithelia exposed to cigarette smoke condensate. Oncogene. 2010;29(25):3650-3664.

65. Xi S, Zhu H, Xu H, Schmidtmann A, Geiman TM, Muegge K. Lsh controls Hox gene silencing during development. Proc Natl Acad Sci U S A. 2007;104(36):14366-14371.

66. Chi SW, Zang JB, Mele A, Darnell RB. Argonaute HITS-CLIP decodes microRNA-mRNA interaction maps. Nature. 2009;460(7254):479-486.

67. Xi S, Geiman TM, Briones V, Guang TY, Xu H, Muegge K. Lsh participates in DNA methylation and silencing of stem cell genes. Stem Cells. 2009; 27(11):2691-2702.

68. Tao Y, Xi S, Briones V, Muegge K. Lsh mediated RNA polymerase II stalling at HoxC6 and HoxC8 involves DNA methylation. PLoS One. 2010; 5(2):e9163.

69. Kemp CD, et al. Polycomb repressor complex-2 is a novel target for mesothelioma therapy. Clin Cancer Res. 2011;18(1):77-90. 